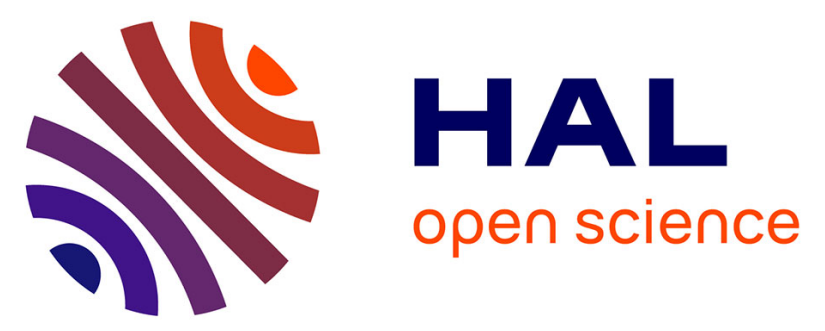

\title{
ARE ULTRA-FAINT GALAXIES AT $z=6-8$ RESPONSIBLE FOR COSMIC REIONIZATION? COMBINED CONSTRAINTS FROM THE HUBBLE FRONTIER FIELDS CLUSTERS AND PARALLELS
}

Hakim Atek, Johan Richard, Mathilde Jauzac, Jean-Paul Kneib, Priyamvada

Natarajan, Marceau Limousin, Daniel Schaerer, Eric Jullo, Harald Ebeling, Eiichi Egami, et al.

\section{- To cite this version:}

Hakim Atek, Johan Richard, Mathilde Jauzac, Jean-Paul Kneib, Priyamvada Natarajan, et al.. ARE ULTRA-FAINT GALAXIES AT $\mathrm{z}=6-8$ RESPONSIBLE FOR COSMIC REIONIZATION? COMBINED CONSTRAINTS FROM THE HUBBLE FRONTIER FIELDS CLUSTERS AND PARALLELS. The Astrophysical Journal, 2015, 10.1088/0004-637X/814/1/69 . hal-01238613

\section{HAL Id: hal-01238613 https://hal.science/hal-01238613}

Submitted on 6 Dec 2015

HAL is a multi-disciplinary open access archive for the deposit and dissemination of scientific research documents, whether they are published or not. The documents may come from teaching and research institutions in France or abroad, or from public or private research centers.
L'archive ouverte pluridisciplinaire HAL, est destinée au dépôt et à la diffusion de documents scientifiques de niveau recherche, publiés ou non, émanant des établissements d'enseignement et de recherche français ou étrangers, des laboratoires publics ou privés. 


\title{
ARE ULTRA-FAINT GALAXIES AT $z=6-8$ RESPONSIBLE FOR COSMIC REIONIZATION? COMBINED CONSTRAINTS FROM THE HUBBLE FRONTIER FIELDS CLUSTERS AND PARALLELS*
}

\author{
Hakim AteK $^{1,2}$, Johan Richard $^{3}$, Mathilde Jauzac ${ }^{4,5}$, Jean-Paul Kneib ${ }^{1,6}$, Priyamvada Natarajan ${ }^{2}$, Marceau Limousin ${ }^{6}$, \\ Daniel Schaerer ${ }^{7,8}$, Eric Jullo ${ }^{6}$, Harald Ebeling $^{9}$, Eitchi Egami $^{10}$, and Benjamin Clement ${ }^{3}$ \\ ${ }^{1}$ Laboratoire d'Astrophysique, Ecole Polytechnique Fédérale de Lausanne, Observatoire de Sauverny, CH-1290 Versoix, Switzerland \\ ${ }^{2}$ Department of Astronomy, Yale University, 260 Whitney Avenue, New Haven, CT 06511, USA \\ ${ }^{3}$ CRAL, Observatoire de Lyon, Université Lyon 1, 9 Avenue Ch. André, F-69561 Saint Genis Laval Cedex, France \\ ${ }^{4}$ Institute for Computational Cosmology, Durham University, South Road, Durham DH1 3LE, UK \\ ${ }^{5}$ Astrophysics and Cosmology Research Unit, School of Mathematical Sciences, University of KwaZulu-Natal, Durban, 4041 South Africa \\ Aix Marseille Université, CNRS, LAM (Laboratoire d'Astrophysique de Marseille) UMR 7326, F-13388, Marseille, France \\ ${ }^{7}$ Observatoire de Genève, Université de Genève, 51 Ch. des Maillettes, 1290, Versoix, Switzerland \\ ${ }^{8}$ CNRS, IRAP, 14 Avenue E. Belin, F-31400, Toulouse, France \\ ${ }^{9}$ Institute for Astronomy, University of Hawaii, 2680 Woodlawn Drive, Honolulu, HI 96822, USA \\ ${ }^{10}$ Steward Observatory, University of Arizona, 933 North Cherry Avenue, Tucson, AZ 85721, USA \\ Received 2015 September 12; accepted 2015 October 8; published 2015 November 17
}

\begin{abstract}
We use deep Hubble Space Telescope imaging of the Frontier Fields to accurately measure the galaxy rest-frame ultraviolet luminosity function (UV LF) in the redshift range $z \sim 6-8$. We combine observations in three lensing clusters, A2744, MACS 0416, and MACS 0717, and their associated parallel fields to select high-redshift dropout candidates. We use the latest lensing models to estimate the flux magnification and the effective survey volume in combination with completeness simulations performed in the source plane. We report the detection of 227 galaxy candidates at $z=6-7$ and 25 candidates at $z \sim 8$. While the total survey area is about $4 \operatorname{arcmin}^{2}$ in each parallel field, it drops to about $0.6-1 \operatorname{arcmin}^{2}$ in the cluster core fields because of the strong lensing. We compute the UV LF at $z \sim 7$ using the combined galaxy sample and perform Monte Carlo simulations to determine the best-fit Schechter parameters. We are able to reliably constrain the LF down to an absolute magnitude of $M_{\mathrm{UV}}=-15.25$, which corresponds to $0.005 L^{\star}$. More importantly, we find that the faint-end slope remains steep down to this magnitude limit with $\alpha=-2.04_{-0.17}^{+0.13}$. We find a characteristic magnitude of $M^{\star}=-20.89_{-0.72}^{+0.60}$ and $\log$ $\left(\phi^{\star}\right)=-3.54_{-0.45}^{+0.48}$. Our results confirm the most recent results in deep blank fields but extend the LF measurements more than two magnitudes deeper. The UV LF at $z \sim 8$ is not very well constrained below $M_{\mathrm{UV}}=-18$ owing to the small number statistics and incompleteness uncertainties. To assess the contribution of galaxies to cosmic reionization, we derive the UV luminosity density at $z \sim 7$ by integrating the UV LF down to an observational limit of $M_{\mathrm{UV}}=-15$. We show that our determination of $\log \left(\rho_{\mathrm{UV}}\right)=26.2 \pm 0.13\left(\mathrm{erg} \mathrm{s}^{-1} \mathrm{~Hz}^{-1} \mathrm{Mpc}^{-3}\right)$ can be sufficient to maintain reionization with an escape fraction of ionizing radiation of $f_{\mathrm{esc}}=10 \%-15 \%$. Future Hubble Frontier Fields observations will certainly improve the constraints on the UV LF at the epoch of reionization, paving the way to more ambitious programs using cosmic telescopes with the next generation of large aperture telescopes such as the James Webb Space Telescope and the European Extremely Large Telescope.
\end{abstract}

Key words: dark ages, reionization, first stars - galaxies: high-redshift - galaxies: luminosity function, mass function - gravitational lensing: strong

\section{INTRODUCTION}

One of the most important challenges in observational cosmology is the identification of the sources responsible for cosmic reionization. Shortly after the big bang, the universe was completely neutral following the recombination of hydrogen atoms, until the first sources started to reionize the neutral gas in their surroundings. Several observational results have now narrowed down the period of reionization to the redshift interval $6<z<12$. Observations of the GunnPeterson effect in the absorption spectra of quasars and

\footnotetext{
* Based on observations made with the NASA/ESA Hubble Space Telescope, which is operated by the Association of Universities for Research in Astronomy, Inc., under NASA contract NAS 5-26555. These observations are associated with programs 13495, 11386, 13389, and 11689. STScI is operated by the Association of Universities for Research in Astronomy, Inc., under NASA contract NAS 5-26555. The Hubble Frontier Fields data and the lens models were obtained from the Mikulski Archive for Space Telescopes (MAST). This work utilizes gravitational lensing models produced by PIs Ebeling, Merten, and Zitrin, funded as part of the HST Frontier Fields program conducted by STScI.
}

gamma-ray bursts indicate that the universe was mostly ionized by $z \sim 6$ (Fan et al. 2006; Chornock et al. 2014). The sensitivity of $\operatorname{Ly} \alpha$ emission to neutral gas is also used to probe the ionization state of the intergalactic medium (IGM) at $z>6$. In particular, the prevalence of Ly $\alpha$ emitters (LAEs) among continuum-selected galaxies (Lyman break galaxies, LBGs) appears to drop very rapidly at $z>6.5$, which suggests an increase in the fraction of neutral hydrogen (Stark et al. 2010; Treu et al. 2013; Pentericci et al. 2014; Schenker et al. 2014). The decline in the LAE fraction also favors a patchy rather than a smooth reionization process. The optical depth of Thomson scattering to the cosmic microwave background recently reported by the Planck collaboration (Planck Collaboration et al. 2015) implies a redshift of instantaneous reionization around $z_{r}=8.8_{-1.2}^{+1.3}$, significantly lower than earlier determinations of $z_{r}=10.6 \pm 1.1$ by the Wilkinson Microwave Anisotropy Probe (Bennett et al. 2013). Despite these major advances, large uncertainties remain regarding the main sources that drive the reionization process. 
Table 1

HST Observations of the HFF Fields

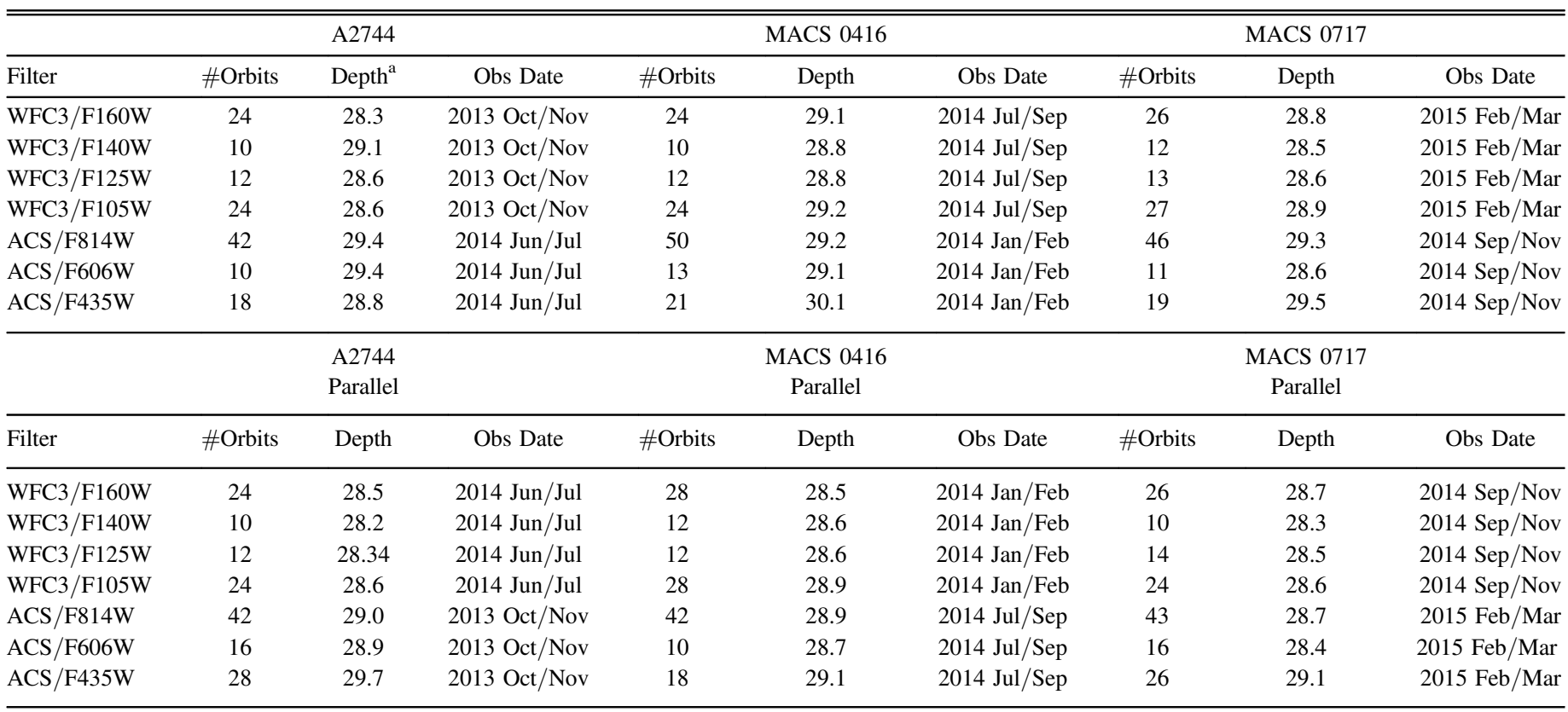

Note.

a The depths of the images are $3 \sigma$ magnitude limits measured in a 0 "! 4 aperture.

Early star-forming galaxies are now thought to be the best candidates for providing the required ionizing power (e.g., Finkelstein et al. 2014; Robertson et al. 2014; Duncan \& Conselice 2015; Mason et al. 2015; Mitchell-Wynne et al. 2015). Deep imaging campaigns of blank fields with the Hubble Space Telescope (HST) and ground-based instrumentation have made important inroads in constraining the galaxy ultraviolet luminosity function (UV LF) out to $z \sim 10$ (e.g., Bunker et al. 2010; Bouwens et al. 2012; Bouwens et al. 2015b; Oesch et al. 2012; McLure et al. 2013; Finkelstein et al. 2014; Schmidt et al. 2014), which, in turn, encodes important information about the cosmic star formation (Bouwens et al. 2015b; Robertson et al. 2015). Indeed, the rest-frame UV radiation traces the recent star formation averaged over the past hundreds of megayears. Therefore, the integration of the UV LF provides the luminosity density of galaxies at a given redshift, which can be translated to the ionizing radiation, assuming a certain star formation history. The UV luminosity density is sensitive to two main parameters: (i) the faint-end slope of the LF, and (ii) the integration limit at the faint end.

The faint-end slope of the LF evolves with redshift and gets as steep as $\alpha \sim-2$ at $z \sim 7$. For comparison, the faint-end slope at $z<0.5$ is very shallow $(\alpha \sim-1.3)$, which indicates a decreasing contribution of faint star-forming galaxies to the total star formation density toward lower redshift (Arnouts et al. 2005; Schiminovich et al. 2005). The predicted evolution of the dark matter halo mass function based on cosmological simulations predicts an even steeper slope of $\alpha \sim 2.3$ of the UV LF at high redshift (Jaacks et al. 2012). The deepest HST observations of blank fields, such as the XDF, put constraints on the faint-end slope down to an absolute magnitude of $M_{\mathrm{UV}} \sim-17.5 \mathrm{AB}$. However, the ability of galaxies to reionize the universe relies on the extent of the steep faintend slope down to lower luminosities, typically around
Table 2

Combined Constraints on the UV LF at $z \sim 7$

\begin{tabular}{lcc}
\hline \hline$M_{\mathrm{UV}}$ & $\log (\varphi)$ & $\varphi_{\mathrm{err}}$ \\
\hline-20.25 & -3.4184 & 0.1576 \\
-19.75 & -3.0263 & 0.1658 \\
-19.25 & -2.9044 & 0.1431 \\
-18.75 & -2.7418 & 0.1332 \\
-18.25 & -2.3896 & 0.1401 \\
-17.75 & -2.1032 & 0.1990 \\
-17.25 & -1.8201 & 0.1940 \\
-16.75 & -1.7548 & 0.1893 \\
-16.25 & -1.6044 & 0.2117 \\
-15.75 & -1.4012 & 0.3123 \\
-15.25 & -1.4012 & 0.3122 \\
\hline
\end{tabular}

Table 3

Number of Galaxy Candidates in Each Field

\begin{tabular}{lcc}
\hline \hline Field & $z=6-7$ & $z=8$ \\
\hline A2744 & 45 & 7 \\
MACS 0416 & 33 & 3 \\
MACS 0717 & 41 & 3 \\
A2744 par & 44 & 3 \\
MACS 0416 par & 33 & 5 \\
MACS 0717 par & 31 & 5 \\
\hline
\end{tabular}

$0.001 L^{\star}$ at $z \sim 7 .{ }^{11}$ While cosmological simulations point to a halo mass limit of $10^{6} M_{\odot}$ for early galaxy formation (Jaacks et al. 2012; Kimm \& Cen 2014; Wise et al. 2014), the depth of current observations, however, limits the exploration of the LF to galaxies brighter than $\sim 0.1 L^{\star}$. One particularly efficient way

\footnotetext{
11 The characteristic magnitude $M_{\mathrm{UV}}^{\star}=-21 \mathrm{AB}$ (e.g., Atek et al. 2014b; Bouwens et al. 2015b).
} 
to push the limits of current facilities is to take advantage of the gravitational lensing offered by massive galaxy clusters, which act as natural telescopes by boosting the flux of background sources (Kneib \& Natarajan 2011).

Since the discovery of the first giant arcs created by strong lensing (Soucail et al. 1987), cosmic lenses have been successfully used to detect intrinsically faint background sources and perform spatially detailed analysis of distant galaxies. Recently, using HST imaging of 25 X-ray-selected clusters in the Abell (Abell et al. 1989) and MACS (Ebeling et al. 2001, 2007, 2010; Mann \& Ebeling 2012) catalogs, the CLASH program (Postman et al. 2012) has made important progress in the characterization of the lensing properties of clusters. Multiwavelength observations have enabled the measurement of the total cluster mass with a precision of $10 \%$ and the detection of some of the most distant galaxies at $z>7$ (e.g., Zitrin et al. 2011; Coe et al. 2013; Bradley et al. 2014). However, two limitations prevented such a program from exploring the faint end of the LF at the epoch of reionization. First, the relatively shallow data compared to the Hubble Ultra-Deep Field (HUDF) restricted the survey to the brighter end of the LF, even in the case of high magnifications. In this sense, wide-area surveys, such as BoRG (Trenti et al. 2011), are indeed well suited to help constrain the brighter part of the LF (Bradley et al. 2012; Schmidt et al. 2014). Second, the lack of very accurate lensing models for some of these clusters, mostly due to the low number of multiple images available to constrain the mass distribution, also thwarts the construction of a reliable LF.

The Hubble Frontier Fields (HFF) project aims at overcoming these two major limitations by obtaining deep multiwavelength observations of six massive galaxy clusters that act as cosmic lenses. The HFF include the deepest optical and near-infrared observations of lensing clusters using HST director discretionary time, which are complemented by a wealth of data, including ALMA, Spitzer, Chandra, XMM, $V L A$, as well as $H S T$ and ground-based imaging and spectroscopic follow-up (e.g., Owers et al. 2011; Ebeling et al. 2014; Grillo et al. 2015; Karman et al. 2015; Medezinski et al. 2015; Ogrean et al. 2015; Rawle et al. 2015; Richard et al. 2015; Rodney et al. 2015; Schirmer et al. 2015; Treu et al. 2015; Zitrin et al. 2015). Based on the full HFF data set of the first cluster A2744, Atek et al. (2015) presented the first constraints on the UV LF at $z \sim 7$ and $z \sim 8$ (see also Ishigaki et al. 2015a). The key result was the steep faint-end slope of $\alpha \sim-2.01$ that extends down to $M_{\mathrm{UV}}=-15.5 \mathrm{AB}$. However, large uncertainties arising from small sample size, lensing models, and cosmic variance still prevent strong conclusions on the total UV luminosity density of galaxies at the epoch of reionization.

In this paper, we combine the complete data set of the three lensing clusters A2744, MACS 0416, and MACS 0717 and their respective parallel fields to search for high-redshift dropout galaxies and put stronger constraints on the UV LF at $z>6$. The paper is organized as follows. In Section 2, we describe the observations. The sample selection method is described in Section 3. In Section 4, we briefly describe the lensing models and the multiple-image identification. The procedure and the results of the computation of the UV LF are presented in Section 5. A summary is given in Section 6. Throughout the paper, we adopt a standard $\Lambda$ CDM cosmology with $H_{0}=71 \mathrm{~km} \mathrm{~s}^{-1} \mathrm{Mpc}^{-1}, \Omega_{\Lambda}=0.73$, and $\Omega_{m}=0.27$ to be
Table 4

The Best-fit $z \sim 7$ Schechter Parameters in Each Individual Field

\begin{tabular}{lccc}
\hline \hline Field & $\begin{array}{c}M_{\text {UV }}^{\star} \\
(\mathrm{AB} \mathrm{mag})\end{array}$ & $\alpha$ & $\begin{array}{c}\log _{10} \phi^{\star} \\
\left(\mathrm{Mpc}^{-3}\right)\end{array}$ \\
\hline A2744 & $-20.92 \pm 0.64$ & $-2.03 \pm 0.13$ & $-3.56 \pm 0.45$ \\
MACS 0416 & $-21.04 \pm 1.10$ & $-2.07 \pm 0.16$ & $-3.67 \pm 0.59$ \\
MACS 0717 & $-21.13 \pm 1.52$ & $-2.02 \pm 0.22$ & $-3.73 \pm 0.83$ \\
A2744 par & $-20.99 \pm 1.05$ & $-2.03 \pm 0.19$ & $-3.62 \pm 0.55$ \\
MACS 0416 par & $-20.94 \pm 1.19$ & $-2.02 \pm 0.20$ & $-3.59 \pm 0.70$ \\
MACS 0717 par & $-20.89 \pm 1.10$ & $-2.00 \pm 0.21$ & $-3.54 \pm 0.62$ \\
\hline
\end{tabular}

consistent with previous studies. All magnitudes are expressed in the $\mathrm{AB}$ system.

\section{HFF OBSERVATIONS}

The HFF clusters and parallel fields were observed by $H S T$ with three ACS (Advanced Camera for Survey) optical filters (F435W, F606W, F814W) and four WFC3 (Wide Field Camera 3) near-IR filters (F105W, F125W, F140W, F160W), as shown in Figure 1. Observations were scheduled in twoepoch sequences, obtaining ACS observations of the main cluster and WFC3 observations of the parallel field in one epoch and swapping instruments in the second epoch (cf. Table 1). We use the high-level science products delivered by the Space Telescope Science Institute (STScI) through the Mikulski Archive for Space Telescopes ${ }^{12}$ (MAST), which include drizzled science and weight images.

Basic calibrations were performed with the standard IRAF procedures CALACS and CALWF3 for ACS and WFC3 data, respectively. Here we chose a pixel scale of 60 mas pixel $^{-1}$ for both optical and IR drizzled images. For the ACS bands we used the "self-calibrated" mosaics, which contain additional corrections applied by the STScI team to better account for charge transfer inefficiency effects. Similarly, the WFC3 bands were also corrected for a time-variable IR background. We refer the reader to a detailed explanation of the data reduction performed by STScI $^{13}$ (A. Koekemoer et al. 2015, in preparation). For the fields that have been observed prior to the HFF program, we combine all the available data using the weight maps included in the HFF data release. Table 2 summarizes the exposure times and the depth achieved in each filter for each of the fields. The limiting magnitude in each filter was calculated using 0 "! 4 diameter apertures randomly distributed in the image to sample the sky variance before fitting the resulting distribution. The quoted depth is given at the $3 \sigma$ level.

\section{SAMPLE SELECTION}

\subsection{Photometric Catalogs}

We constructed the photometric catalogs in each field using the SExtractor software (Bertin \& Arnouts 1996). We first matched all the images to the same point-spread function (PSF) using a model based on the largest PSF derived with TinyTim (Krist et al. 2011). In order to increase the sensitivity to faint sources, we created deep images by using inverse variance (IVM) weight maps to combine all IR frames for $z \sim 7$ sources

\footnotetext{
12 https://archive.stsci.edu/prepds/frontier/

13 http://www.stsci.edu/hst/campaigns/frontier-fields/
} 
and F125W, F140W, and F160W for $z \sim 8$ sources, respectively. This deep image is used for source detection in the SExtractor dual-image mode, while individual images are used for photometry, weighted by the individual IVM images.

One important limitation for the detection of the faintest sources in the cluster fields is the contamination from the intracluster light (ICL) in the central region of the cluster. This diffuse light, concentrated primarily in the central region of the cluster, is due to the brightest cluster galaxy (BCG) or other bright cluster members and the tidal stripping of stars from interacting galaxies during the merging history of the cluster (e.g., Montes \& Trujillo 2014). In order to mitigate the contamination of sources close to the cluster center, we subtract a median-filtered image from the detection one with a filter size of $\sim 2^{\prime \prime} \times 2^{\prime \prime}$, while the photometry is performed on the original image. The adopted filter size is a trade-off between the removal of extended bright emission and the appearance of artifacts close to the bright galaxies of the cluster core. We note that the median-filtered detection image allows us to detect five more sources on average in cluster fields compared to the original images. This is also important for the visual inspection of galaxy candidates as the background flux is much lower in these corrected images.

In order to estimate the total flux errors, we ran simulations of galaxies with different sizes and profiles (see the completeness simulations in Section 5.1) and compared the recovered fluxes to the input values. We find that the median-filtering approach achieves an uncertainty of $0.5 \mathrm{mag}$ for the faintest sources $\left(H_{140} \sim 28-29 \mathrm{mag}\right)$, by using a local background estimate with back_size $=6$ in SExtractor for photometry. In addition, the following extraction parameters were set to improve the detection of the faintest sources in the field: detect_minarea $=2$ and detect_thresh $=1.5$ for the detection and deblend_nthresh $=16$ for source deblending. Two types of photometry are used throughout this work. The isophotal magnitude (ISO) is used to compute the colors and ensure that the same aperture is used across the filter set. The total flux is measured within the Kron radius using the AUTO magnitude, which hereafter is used as the total magnitude. We modified the magnitude errors to account for pixel-to-pixel noise correlations in the drizzled images following Casertano et al. (2000). Finally, the individual catalogs were matched into a master photometric catalog and cleaned from spurious sources.

\subsection{High-redshift Dropout Selection}

We adopted the Lyman break selection technique (Steidel et al. 1996; Giavalisco 2002) to detect high-redshift galaxy candidates. The selection is based on color-color criteria to sample the UV continuum dropout of the IGM absorption blueward of Ly $\alpha$ caused by the intervening hydrogen along the line of sight and minimize contamination by low-redshift red objects at the same time. Following Atek et al. (2015), we use the following criteria to select $z=6-7$ galaxies:

$$
\begin{aligned}
& \left(I_{814}-Y_{105}\right)>1.0 \\
& \left(I_{814}-Y_{105}\right)>0.6+2.0\left(Y_{105}-J_{125}\right) \\
& \left(Y_{105}-J_{125}\right)<0.8 .
\end{aligned}
$$

In addition, we require all sources to be detected in the deep IR image and in at least two IR bands with $4 \sigma$ significance or higher. We also reject any galaxy that shows up at a significant

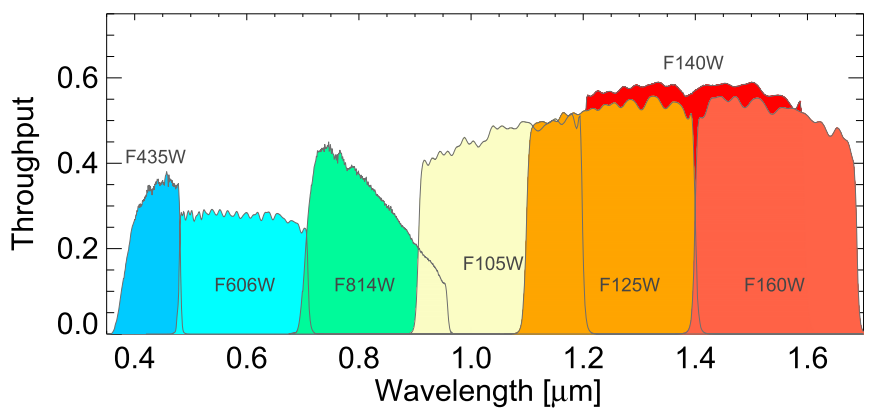

Figure 1. Throughput curves of the HST/ACS and WFC3 filter set used for the HFF observations.

(at $1.5 \sigma$ ) level in the deep optical combination of $B_{435}+V_{606}$ images. To preserve the Lyman break criterion in the case of nondetection in the $I_{814}$ image, we assign a $2 \sigma$ limiting magnitude to this band. Therefore, we select only galaxies with $Y_{105}$ at least 1 mag brighter than the $I_{814}$ depth. Similarly, we select $z \sim 8$ galaxies that satisfy

$$
\begin{aligned}
\left(Y_{105}-J_{125}\right) & >0.5 \\
\left(Y_{105}-J_{125}\right) & >0.3+1.6\left(J_{125}-H_{140}\right) \\
\left(J_{125}-H_{140}\right) & <0.5 .
\end{aligned}
$$

We require a $4 \sigma$ detection in a deep $J_{125}+H_{140}+H_{160}$ image and the detection in the stacked deep optical image (four ACS filters) to be less than $1.5 \sigma$. The deep optical image is about one magnitude deeper than the faintest sources (detected in the IR) accepted in our sample. In Figure 2 we show the result of our selection procedure. The green circles show the location of all high-redshift galaxies identified in this work in the color-color diagram. The dotted and solid lines represent the color evolution of low-redshift elliptical galaxies and highredshift starbursts, respectively, constructed from Coleman et al. (1980) and Kinney et al. (1996) templates. An attenuation of $A_{V}=1,2,3$ is applied to the blue, orange, and red curves, respectively. The shaded region represents our adopted selection window, which was chosen to minimize contamination from low-redshift interlopers and red objects such as cool stars represented by magenta points. Our final sample, combining cluster and parallel fields, contains 227 galaxies at $z \sim 6-7$, according to the selection based on Equation (1), and 25 galaxies at $z \sim 8$ based on the selection of Equation (2) (see Table 3 ). We find roughly the same number of candidates in the cluster and the parallel fields. While the survey area is smaller in the lensed field, the magnification bias balances the number density, allowing the detection of much fainter galaxies than in the parallel fields. The redshift distribution of the selected candidates and the redshift selection function at $z \sim 7$ and $z \sim 8$ are shown in Figure 3.

\subsection{Sources of Contamination}

We now discuss the main sources of contamination for our high-redshift sample of galaxies. A first possibility is spurious sources produced by detector artifacts, diffraction features, or photometric errors scattering into the color selection space. We have visually inspected all of our candidates to identify such contaminants. Most of the spurious sources were already cleaned using the weight maps that exclude the frame edges and the IR blobs identified by STScI calibrations. We find that the remaining artifacts are mostly diffraction spikes of bright 
stars and few very bright cluster galaxies in the field. Fake sources due to photometric noise are also minimal since we require the detection in at least two different bands.

The second potential source of contamination are lowredshift galaxies that show similar colors to high-redshift candidates. According to our stringent spectral break criteria, such sources need to show an extremely red continuum or a large Balmer/4000 A break. Dust-obscured galaxies would be excluded by our selection because they would exhibit relatively red colors redward of the break. The remaining galaxies that can enter the selection need to have a large Balmer break and relatively blue continuum at longer wavelengths. The existence of these peculiar objects has been discussed in Hayes et al. (2012), where a spectral energy distribution (SED) of a young burst superimposed on an old stellar population with an extreme $4000 \AA$ A break can mimic the Lyman break colors. Such objects should be very rare and represent only a minor contamination. Moreover, in the case of a significant contamination by these interlopers, we expect to detect the blue continuum by stacking the optical images of our candidates, which is not the case.

Alternatively, the Lyman break colors can also be mimicked by extremely strong emission lines in $z=1-3$ star-forming galaxies (e.g., Atek et al. 2011, 2014a; van der Wel et al. 2011). In this class of galaxies the contribution of the emission-line flux to the total broadband flux is about $25 \%$ on average and can reach $85 \%$ (Atek et al. 2011) and can have important implications, not only for the high-redshift galaxy selection but also for the age and stellar mass derived from SED fitting (Schaerer \& de Barros 2009; Wilkins et al. 2013; Schenker et al. 2014; Huang et al. 2015; Pénin et al. 2015). In our case, the contamination of one band can lead to an artificial Lyman break, because the faint continuum remains undetected blueward of the break. Atek et al. (2011) estimate that the optical data should be about 1 mag deeper than the detection band to be able to rule out such interlopers. Our criteria impose a minimum break of 0.8 mag between the object flux and the limiting magnitude of the optical band. Moreover, such a contamination should be even smaller because our stacked candidate images, which are at least $1.3 \mathrm{mag}$ deeper than the candidate's flux, show no significant detection in the optical bands shortward of the break.

In general, observationally, estimating the contamination rate of such sources proves very difficult at $z>6$ because it hinges on large spectroscopic follow-up programs that aim at detecting the redshifted $\operatorname{Ly} \alpha$ emission line in these galaxies. In addition to the large amount of telescope time needed to reach the required depth, the absence of Ly $\alpha$ emission does not exclude the high-redshift nature of the associated objects. Indeed, the increasing neutral hydrogen fraction at $z>6$ easily absorbs and diffuses Ly $\alpha$ photons (Atek et al. 2009; Stark et al. 2010; Caruana et al. 2014; Schenker et al. 2014). Therefore, one must rely on simulated colors based on galaxy spectral templates to estimate the contamination rate of low-redshift galaxies, which thus has been found to be less than $10 \%$ in most studies (e.g., Oesch et al. 2010; Bouwens et al. 2015b).

Another source of contamination to consider are low-mass stars. As we can see in Figure 2, a significant fraction of these stars (shown in magenta) can have similar colors to highredshift galaxies. However, we have excluded any source that has a SExtractor stellarity parameter greater than 0.8 to minimize point-like objects in our sample. Also, the number density of cool stars ranges from 0.02 to $0.05 \operatorname{arcmin}^{2}$ derived from observations with similar or better depths (Bouwens et al. 2015b). This translates to about one contaminant in the entire $z \sim 7$ and $z \sim 8$ galaxy samples. We also consider transient events as possible contaminants. Because observations are taken at two different epochs (see Table 4), a supernova explosion may appear in IR images and not in the optical ones and could be selected as a dropout candidate. Similar colors can also be obtained in the case where the IR data were taken first and the supernova faded until the optical images. Again, different reasons point toward a negligible contamination level from transient sources. Such objects would show point-like profiles that would be excluded by the stellarity criterion explained above and our additional visual check for unresolved sources. The detection rate of supernovae is also very small, and so far none of the supernova detections in the dedicated search program of the HFF (e.g., Rodney et al. 2015) have been inadvertently selected as high- $z$ galaxies in the different HFF studies (Atek et al. 2014b, 2015; Finkelstein et al. 2014; Ishigaki et al. 2015a).

\section{HFF CLUSTER MASS MODELS}

In order to exploit the full potential of the HFF cluster lenses, we first need a robust model describing the total mass distribution and the lensing properties. In an effort to provide the community with all the required lensing maps to interpret background source observations, several groups have submitted their models prior to the HFF (e.g., Bradač et al. 2005; Merten et al. 2011; Johnson et al. 2014; Richard et al. 2014; Coe et al. 2015; Grillo et al. 2015). With the availability of new deep HST, Spitzer, and spectroscopic observations, the models were significantly improved thanks in particular to the discovery of a large number of multiple images (Diego et al. 2015; Jauzac et al. 2014, 2015; Zitrin et al. 2014; Grillo et al. 2015; Ishigaki et al. 2015a; Wang et al. 2015). Most notably, using a set of $\sim 180$ and $\sim 200$ multiple images in A2744 and MACS 0416, respectively, Jauzac et al. (2014, 2015) reconstructed the projected cluster mass down to a precision of $\sim 1 \%$, which represents a significant improvement over pre-HFF mass models.

In the present study, we use the most recent cluster models based on full-depth HFF observations and constructed by the CATS (Clusters As Telescopes) team. The models developed for the first two HFF clusters, namely, A2744 and MACS 0416, are already available on the MAST archive. However, STScI has started a new mass mapping initiative so all the teams provide HFF mass models of both clusters taking advantage of the full depth of the HFF data, but using the same inputs. These "unified" models will be submitted to STScI as part of our answer to the HFF call for the community to provide updated lensing maps. ${ }^{14}$ The mass modeling of the third cluster MACS 0717 will be presented in detail in Limousin et al. (2015).

The mass reconstruction of each of the clusters is explained in the publications listed above. Here we briefly describe the key points of the mass modeling procedure.

The CATS HFF mass models are built using the Lenstool ${ }^{15}$ software (Kneib 1993; Jullo et al. 2007; Jullo \& Kneib 2009). For the strong-lensing analysis, we are using a parametric approach that consists of modeling the cluster mass distribution

\footnotetext{
14 https://archive.stsci.edu/prepds/frontier/lensmodels /

15 http://projets.lam.fr/projects/lenstool/wiki
} 

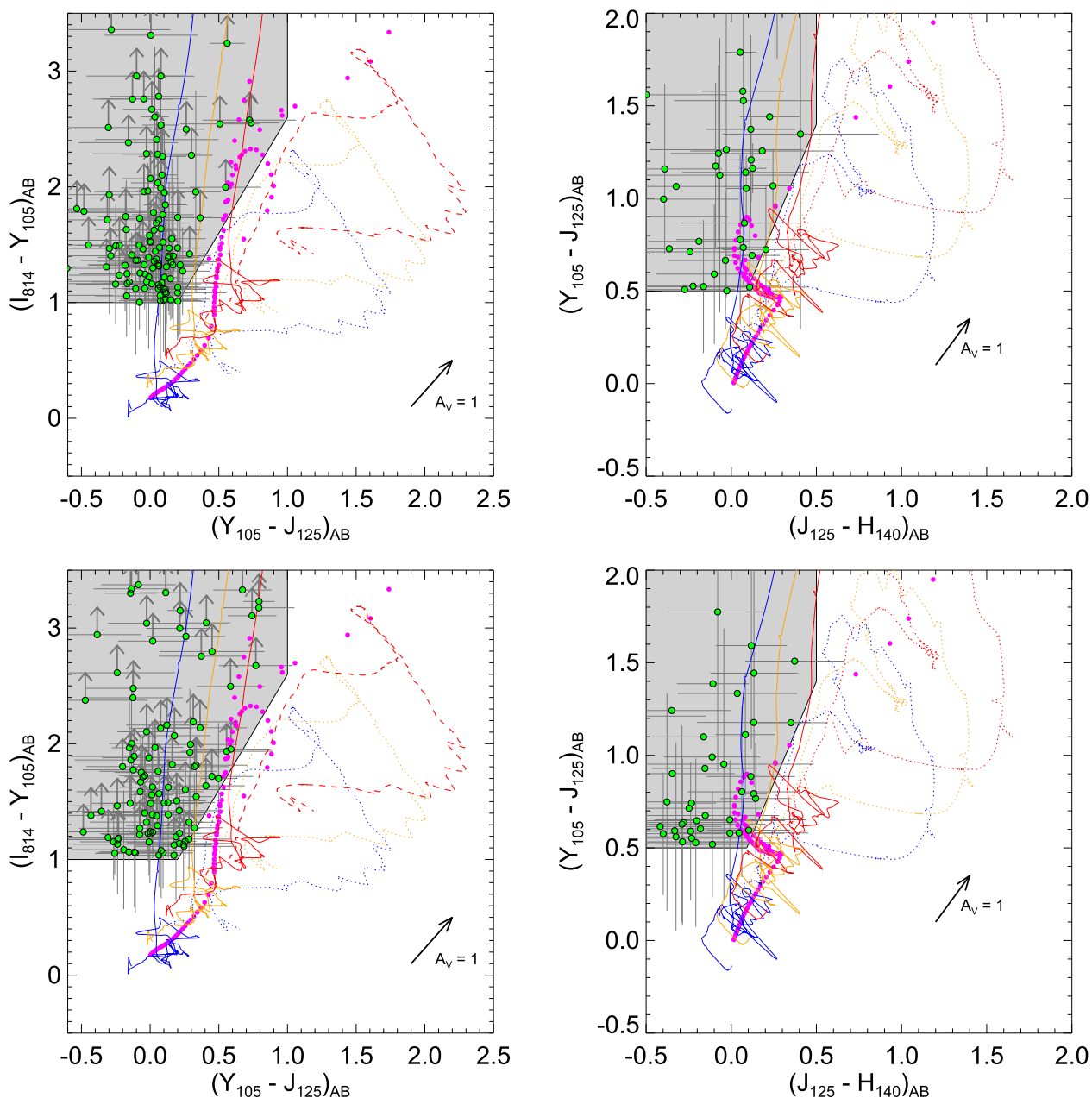

Figure 2. Color-color selection windows (represented by the shaded regions) of high- $z$ dropout candidates. The left and right panels show the selection of $z \sim 7$ and $z \sim 8$ galaxies, respectively. The top panels are for cluster fields and bottom panels for parallel fields. The green circles (with associated $1 \sigma$ uncertainties) represent a compilation of all galaxies satisfying our selection criteria and included in our $z>6$ samples. The dotted lines show the color track, i.e., the redshift evolution of colors, of low-redshift elliptical galaxies generated from Coleman et al. (1980) templates, whereas solid lines show starburst galaxies generated from Kinney et al. (1996) templates. The color code, from blue to red, illustrates the impact of extinction in steps of $A_{V}=1$. Finally, the magenta points denote the color track of stars generated from Chabrier et al. (2000) templates.

using both cluster-scale and galaxy-scale halos. Galaxy-scale halos are important in the mass modeling, as multiple image configurations are impacted by their location. Indeed, if a multiple image is close to a cluster galaxy, then the distortion created by the lensing effect will be a combination of both the cluster potential itself and a galaxy-galaxy lensing effect due to the potential of the cluster member. The potentials describing these components are modeled using pseudo-isothermal elliptical mass distribution (Elíasdóttir et al. 2007).

For MACS J0416, we used 149 of the most secure multiple images (over the 194 identified) to constrain the mass model. Our best-fit mass model of the cluster inner core consists of two cluster-scale halos, well aligned with the light peaks from the two BCGs, plus 98 galaxy-scale halos, corresponding to the BCGs. For Abell 2744, we used 154 most secure multiple images (over the 181 identified). Our best-fit mass model consists of two cluster-scale halos to describe the dark matter distribution on large scale, combined with 733 galaxy-scale halos, describing the distribution of cluster galaxies. Both models delivered really good errors in the predictions of multiple image positions, an rms of 0." 68 and 0".79 for MACS J0416 and Abell 2744, respectively. For MACS J0717, we used the 140 most secure multiple images (over 163 identified) to constrain a mass model composed of four large-scale DM halos, plus 92 galaxy-scale halos, corresponding to the BCGs. More details of the strong lensing (SL) analysis will be given in Limousin et al. (2015).

\subsection{Multiple Images}

In addition to flux magnification, strong lensing produces multiple images of the same background galaxy. Therefore, we need to identify multiple images to be removed from the galaxy number counts before computing the UV LF. For each galaxy, we predict the position of potential counterimages by using the mass model and Lenstool to project its position into the source plane before lensing back the source into the image plane, where Lenstool predicts the position of all the multiple images. In the vicinity of these positions, we look for dropout sources that show similar colors and photometric redshifts. In the case of well-resolved sources, we also verify that they have similar morphological and geometrical symmetries constrained by the lensing model. In A2744 we find three systems at $z \sim 7$, with a total of nine multiple images, which were discussed in Atek 


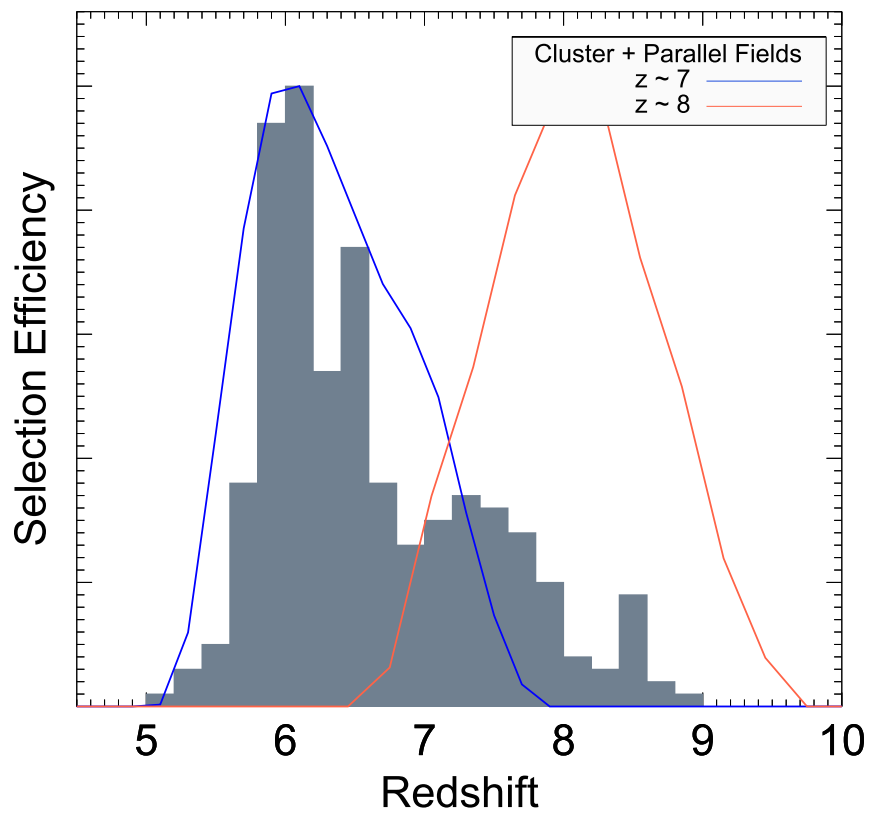

Figure 3. Redshift selection function for all the fields at $z \sim 7$ and $z \sim 8$. The curves are the result of our completeness simulations in recovering the input sources marginalized over the redshift, while the shaded histograms represent the actual selected galaxies in our sample. The median redshift of the our $z \sim 8$ is significantly lower than expected owing to the depth of the observations and the redshift evolution of the LF.

et al. (2014b). In MACS 0416, we identify seven systems at $z \sim 7$

In the case of MACS 0717, the multiple image region extends beyond the WFC3 field of view. Therefore, we expect each of the galaxy candidates to have counterimages with similar flux/magnification ratios given the lensing geometry of this cluster. The uncertainty on the image position predicted from the lensing model of MACS 0717 is about rms $=1.9$ arcsec, much larger than in the two other clusters (see Limousin et al. 2015). Moreover, the shape of the critical line is not as well constrained, which makes it harder to identify multiple-image systems. From the Lenstool model, we estimate the multiplicity in MACS 0717 to be three on average in the WFC3 field of view. Therefore, we divide the number of galaxy candidates by this multiplicity to obtain the final number counts used for the LF calculation.

\section{THE GALAXY UV LUMINOSITY FUNCTION}

We now turn to computing the galaxy UV LF at $z \sim 7$ and $z \sim 8$ by combining the high- $z$ candidate samples from all the fields. For each field we calculate the completeness function and the effective survey volume to derive the LF. While in the parallel fields we proceed with standard completeness simulations widely used in analyzing blank-field surveys, we need to take into account the lensing effects in the cluster fields.

The primary goal of the HFF program is to extend the current limits of deep galaxy surveys by using strong lensing to magnify intrinsically faint sources behind the galaxy clusters. In parallel, the survey area in the source plane is significantly reduced for high magnifications. Consequently, the efficiency of a given lensing cluster in probing the high-redshift universe, which can be quantified by the number of magnified sources

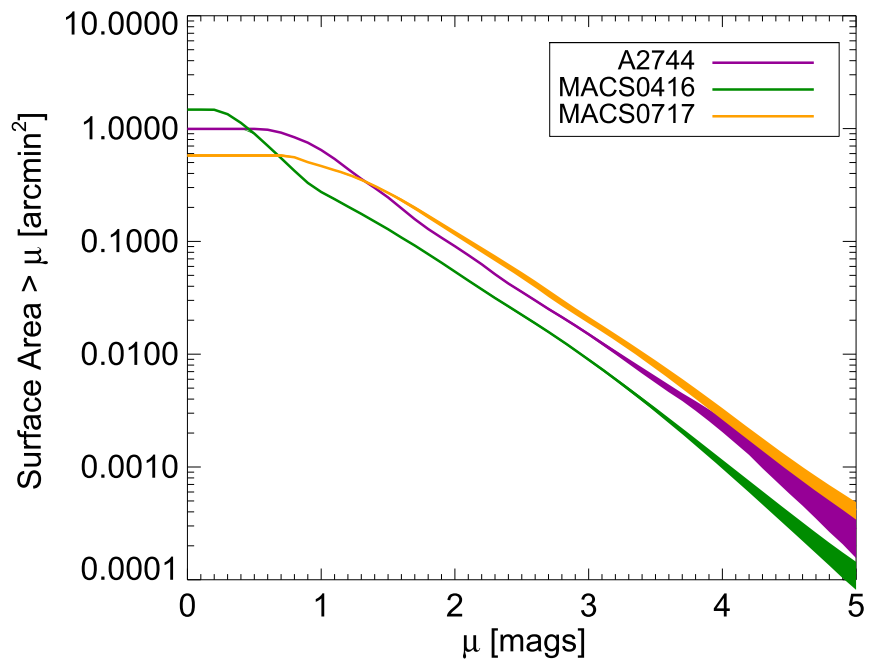

Figure 4. Cumulative surface area in the source plane at $z \sim 7$ as a function of the amplification factor (in magnitudes) derived from the mass modeling of the three HFF clusters. Uncertainties in the surface area are also shown at the $1 \sigma$ level.

discovered, is the result of a trade-off between the amplification factor $\mu$ and the source plane area $\sigma$. We show in Figure 4 the cumulative survey area as a function of the amplification factor.

While the survey area in the image plane corresponds to the WFC3 field of view, i.e., about $4.7 \operatorname{arcmin}^{2}$, we can see that the total survey area is reduced to about $0.6-1 \operatorname{arcmin}^{2}$ in cluster fields. Wong et al. (2012) define the cluster magnification power as the cross section for magnifying a source above minimum threshold of $\mu=3$ (see also Richard et al. 2014). Although the precise value of the threshold is somewhat arbitrary, the number of high-redshift detections in our galaxy samples peaks around $\mu=3$, or $\mu \sim 1.2$ mag (see the top panel of Figure 5). For this typical value, the total survey area drops to values of around $0.2-0.5 \mathrm{arcmin}^{2}$ for the three clusters of this study.

The parallel fields are located about 6 arcmin away from the cluster lenses with a small yet nonnegligible magnification. It is important to assess the lensing effects on the UV LF, both on the magnitude and on the survey volume for these regions. Among the different lensing models available in the HFF project, only Merten et al. (2011) provide a wide-field magnification map for the three clusters that covers their flanked fields. We used the magnification maps at $z \sim 9$ that have a resolution of 25 arcsec pixel ${ }^{-1}$ to estimate the flux amplification of galaxies. Since no deflection map is available, the volume reduction is estimated by dividing the area by the amplification map. The harmonic mean of the amplification factor ranges from 1.11 to 1.23 in the three fields, with typical errors of about $10 \%$. The inclusion of the lensing effects introduces only small changes in the UV LF, within the error bars, basically shifting $M_{\mathrm{UV}}$ to slightly fainter values and $\phi$ to higher values.

We now describe how we estimate the effective survey volume for each cluster by combining the source plane area with the redshift selection function and the recovery rate of simulated galaxies as a function of different galaxy and lensing parameters. 


\subsection{Completeness Simulations}

Following Atek et al. (2015), we run extensive Monte Carlo simulations to assess the completeness level as a function of the intrinsic magnitude. A total of 10,000 galaxies were simulated for each of the six fields. We take the galaxy profile into account by creating two samples of exponential disks and de Vaucouleur shapes (Ferguson et al. 2004; Hathi et al. 2008). These galaxy profiles are then distorted by gravitational lensing according to our mass model. As for galaxy sizes, we adopt a lognormal distribution with a mean half-light radius of 0 "! 15 and sigma $=0$ ".07. For consistency with previous results (Bouwens et al. 2004; Ferguson et al. 2004; Hathi et al. 2008; Oesch et al. 2010, 2014; Grazian et al. 2011, 2012; Huang et al. 2013), the distribution is based on the sizes derived from spectroscopically confirmed LBG samples at $z \sim 4$ (Vanzella et al. 2009), while accounting for a redshift evolution of the intrinsic physical size of galaxies with a factor of $(1+z)^{-1}$. HST observations of dropout galaxies at $z>6$ redshifts also reveal small sizes of less than 0"! 3 (Mosleh et al. 2012; Ono et al. 2013), and smaller for galaxies fainter than $M_{\mathrm{UV}}-21 \mathrm{mag}$. More recently, Kawamata et al. (2015) measured the size of lensed dropout galaxies in the HFF cluster A2744 and reported similar results. Small sizes around 0!' 1 have also been observed in lensed galaxies by Coe et al. (2013) and Zitrin et al. (2014). In addition, based on the results of Huang et al. (2013), we adopt a size-luminosity relation $r \propto$ $L^{\beta}$, with $\beta=0.25$, for our simulations (see also Mosleh et al. 2012; Kawamata et al. 2015).

After assigning random redshifts in the range [5.5, 7.5], we simulate galaxy magnitudes in each HST band using starforming SED templates from Kinney et al. (1996). In this step, we also assign random intrinsic absolute magnitude (in the restframe UV) in the range $M_{\mathrm{UV}}=[-14,-24] \mathrm{mag}$. We then include 10 simulated galaxies in the actual images of each band for a total 1000 images. This is where the cluster and parallel fields are treated differently in the simulations. In the cluster fields, galaxies are not included directly in the images but are simulated in the source plane. They are lensed into the image plane using the corresponding mass model. This way we ensure that all the lensing effects, including magnification, shape distortion, and position relative to the critical line, are fully taken into account. Finally, for all the images, we run the same procedure used to select high- $z$ galaxies and determine the completeness function, which represents the fraction of the original galaxies recovered in our selection as a function of the parameters described above. This completeness function is in turn incorporated in the computation of the effective volume in each magnitude bin following the equation

$$
V_{\mathrm{eff}}=\int_{0}^{\infty} \int_{\mu>\mu_{\min }} \frac{d V_{\mathrm{com}}}{d z} f(z, m, \mu) d \Omega(\mu, z) d z,
$$

where $\mu_{\min }$ is the minimum amplification factor $\mu_{\text {min }}$ required to detect a galaxy with a given apparent magnitude $m$ and $V_{\text {com }}$ is the comoving volume; $f(z, m, \mu)$ is the completeness function that depends on the redshift $z$, apparent magnitude $m$, and amplification factor $\mu$, and $d \Omega(\mu)$ is the area element in the source plane, which is a function of magnification and redshift.

Figure 6 presents the results of our effective volume estimates in each field, marginalized over the intrinsic absolute UV magnitude. The extent of each filled region represents the $68 \%$ confidence intervals. We can clearly see the importance of
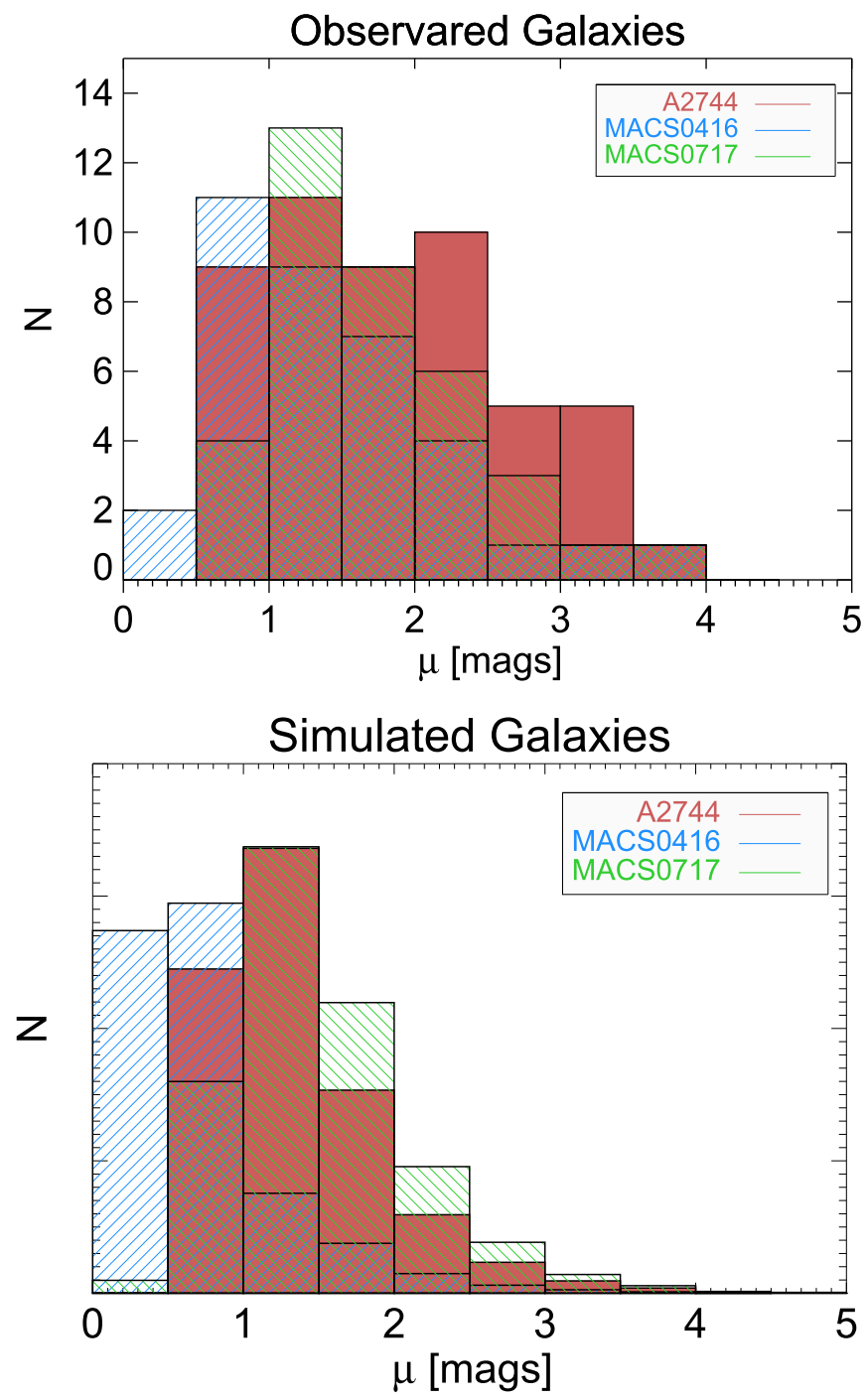

Figure 5. Distribution of the amplification factor, expressed in magnitudes. Top: distribution for the $z \sim 7$ candidates in each of the lensing clusters. Bottom: result of the completeness simulations in the three cluster cores, which contain about 80,000 objects each.

gravitational lensing in extending the survey depth to fainter galaxies. While the blank-field completeness drops abruptly before $M_{\mathrm{UV}}=-18 \mathrm{mag}$, it becomes shallower in cluster fields and extends down to $M_{\mathrm{UV}}=-15 \mathrm{mag}$, although at a level of $10 \%$ or less.

\subsection{The UV LF at $z=6-7$}

Using the derived effective volume as a function of absolute magnitude, we compute the UV LF following the equation

$$
\phi(M) d M=\frac{N_{i}}{V_{\mathrm{eff}}\left(M_{i}\right)},
$$

where $N_{i}$ and $M_{i}$ are the number of galaxies and the absolute magnitude, respectively, in each magnitude bin. Following most of the studies in the literature, we choose a bin size of $0.5 \mathrm{mag}$, whereas the magnitude varies from one field to another. The individual LF determinations in each field are presented in Figure 7. The top panels show the results in the cluster fields, while the bottom panels are for parallel fields. 

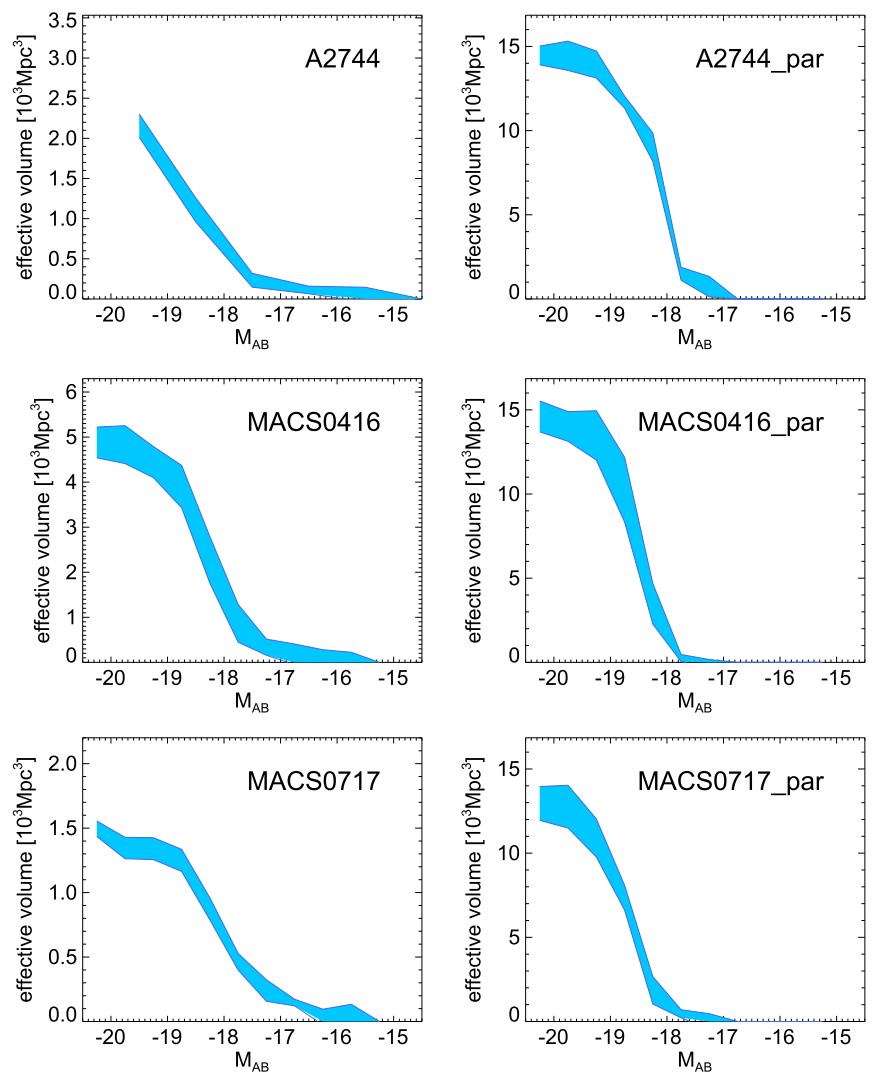

Figure 6. Effective survey volume as a function of absolute UV magnitude for the redshift $z \sim 7$ sample. Each curve is based on our completeness simulations for each cluster or parallel field. The total area for the cluster fields is corrected for lensing effects, whereas the blank-field area is based on the full WFC3 field of view (see text for a detailed explanation). The blue region represents the $95 \%$ confidence intervals of the completeness estimate.

The LF extends to $M_{\mathrm{UV}}=-18.25$ in blank fields, whereas it reaches a magnitude of $M_{\mathrm{UV}}=-15.25$ in cluster fields thanks to the lensing magnification. This gain can already be seen in the completeness function results (see Figure 5), reaching fainter magnitudes in cluster fields. Figure 6 also shows the distribution of the amplification factor for the dropout samples selected behind the three lensing clusters. The flux amplification is essentially in the range $\mu \sim 1.25-75$, with a median value of 5.2, 3.9, and 4.9 in A2744, MACS 0416, and MACS 0717, respectively.

In addition to the sample contamination discussed in Section 3.3, several sources contribute to the uncertainties of the LF data points. The mass model errors, affecting the magnification and the survey volume (or the source-plane area $\Omega$ ), are propagated into the LF determination in the case of cluster fields. We also include Poisson errors and the cosmic variance estimate based on the recent results of Robertson et al. (2015) for both cluster and parallel fields. Finally, uncertainties from incompleteness simulations, as seen in Figure 5, are incorporated in the final LF. The bright end is dominated by cosmic variance errors and small number counts, while the faint-end error bars reflect mostly the large incompleteness uncertainties at these faint magnitudes and small statistics.

We determine the shape of the rest-frame UV LF by fitting a Schechter function (Schechter 1976) to our data, which has been extensively used to describe the galaxy UV LF across a wide redshift range (e.g., Bunker et al. 2004; Beckwith et al. 2006; Bouwens et al. 2006; McLure et al. 2009; Ouchi et al. 2009; Reddy \& Steidel 2009; Wilkins et al. 2010; Oesch et al. 2012; Willott et al. 2013). The Schechter function can be expressed in terms of absolute magnitudes as

$$
\phi(M)=\frac{\ln (10)}{2.5} \phi^{\star} 10^{0.4(\alpha+1)\left(M^{\star}-M\right)} \exp \left(-10^{0.4\left(M^{\star}-M\right)}\right) .
$$

We perform a Schechter fit to the LF in each field and find a faint-end slope $\alpha$ between -2.0 and -2.07 (see Equation (5)). The best-fit Schechter parameters are shown in Table 4. We include in the fit the data points from Bouwens et al. (2015b, black squares) to constrain the bright end of the LF. The faintest bin is reached in A2744 at $M_{\mathrm{UV}}=-15.25$. There is a hint of a shallower slope at those magnitudes that could be the result of large uncertainties in the completeness estimate, which is typically less than $10 \%$ in this bin. As a matter of fact, a similar decline is observed in MACS 0416 around $M_{\mathrm{UV}}=-16.25$, whereas the slope is constantly steep at these magnitudes in A2744. It is clear that the constraints on the LF in MACS 0717 are not as good as in the other cluster fields. This is likely the result of larger uncertainties on the lensing model due to the complex structure of the galaxy cluster. For instance, the model prediction for image position has an rms error of $\sim 1.9$ arcsec, while it is is about $0.7-0.8$ arcsec in MACS 0416 and A2744. These kinds of deviations in the LF data points can also be observed in A2744 when using an old model based on pre-HFF observations that similarly yielded large uncertainties. Nonetheless, the overall shape of the LF remains consistent with the other clusters' results.

We now combine all the LF constraints from the lensed and parallel fields to compute the most robust UV LF at $z \sim 7$. The result is shown in Figure 8. Together with our data points, we show the most recent results from the literature from the blank fields as described in the legend. We ran MCMC simulations with $10^{6}$ realizations to find the best fit to the LF and estimate the uncertainties on the Schechter parameters. We find that the UV LF at $z \sim 7$ has a faint-end slope of $\alpha=-2.04_{-0.17}^{+0.13}$, a characteristic $M^{\star}=-20.89_{-0.72}^{+0.60}$, and $\log \left(\phi^{\star}\right)=-3.54_{-0.45}^{+0.48}$. This is in excellent agreement with earlier results presented in Atek et al. (2015), where we computed the UV LF in the HFF cluster A2744. In Figure 9, we show the likelihood analysis of the Schechter parameters at $z \sim 7$, marginalized over two parameters at a time. Our results are in good agreement with the most recent results reported from the blank fields (see Table 5). In particular, the faint-end slope is close to the determination of Finkelstein et al. (2014) with $\alpha=-2.03_{-20}^{+21}$ and Bouwens et al. (2015b) with $\alpha=-2.03 \pm 0.14$. Our results show only a slightly steeper faint-end slope than the values reported in the Hubble extreme deep field (McLure et al. 2013; Schenker et al. 2013a) with $\alpha=1.90_{-0.15}^{+0.14}$ and $\alpha=1.90_{-0.15}^{+0.14}$, respectively, and agree within the reported uncertainties. Also using the gravitational lensing of the first HFF cluster A2744, Ishigaki et al. (2015a) find a slightly shallower slope of $\alpha=-1.94_{-0.10}^{+0.09}$, which is still in agreement with our value within the errors. We also performed a Schechter fit while excluding data points from the MACS 0717 cluster, which yields very similar parameters: $\alpha=-2.03, \quad$ a characteristic $M^{\star}=-20.86, \quad$ and $\log$ $\left(\phi^{\star}\right)=-3.52$. Importantly, we note that the uncertainties on the faint-end slope decrease to $\sigma_{\alpha} \sim 0.1$. 

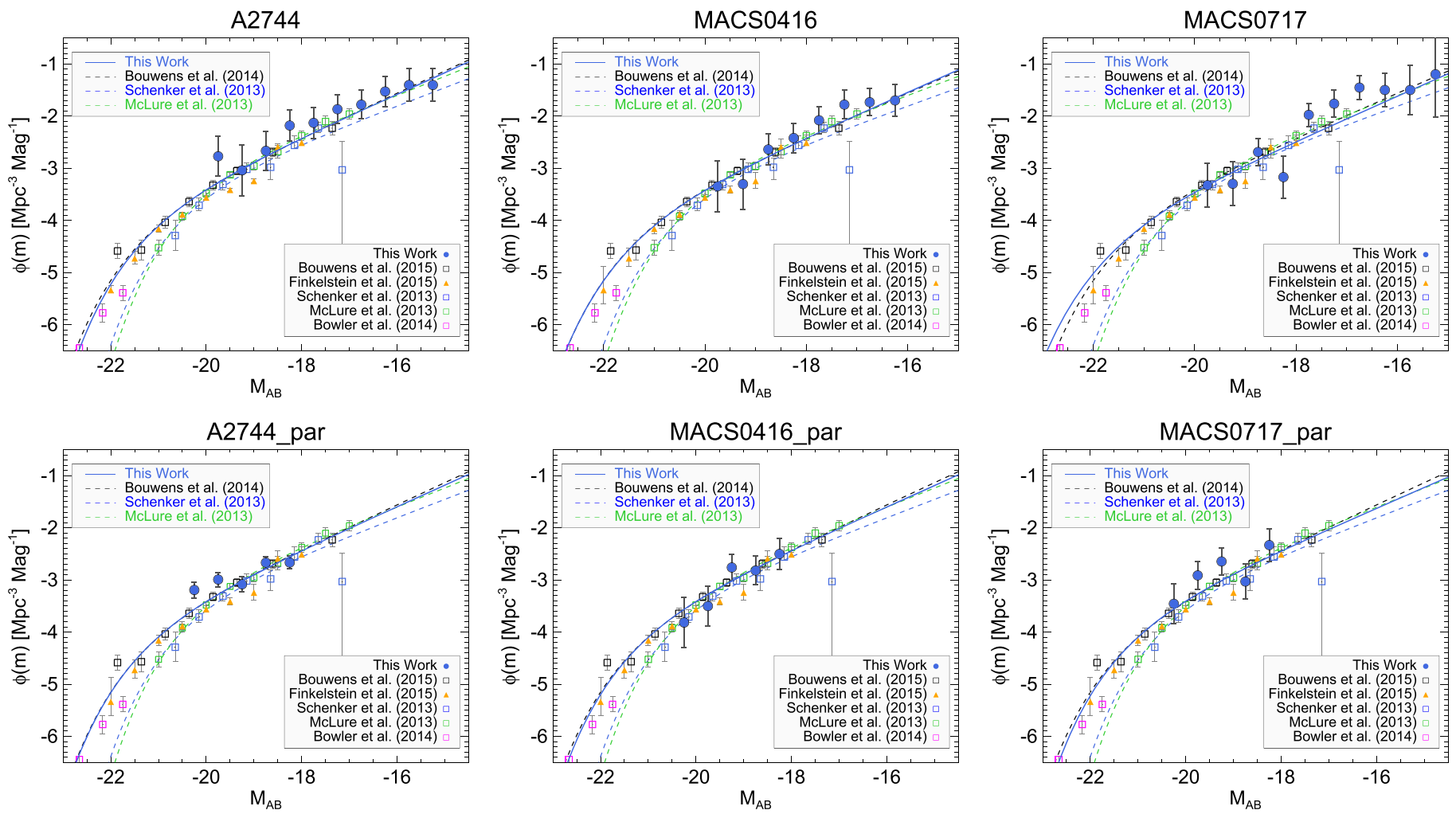

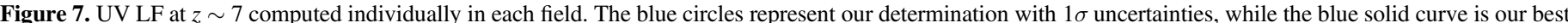

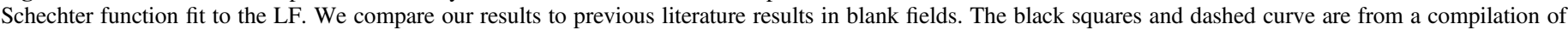

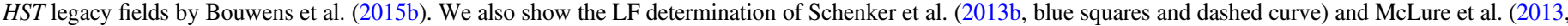

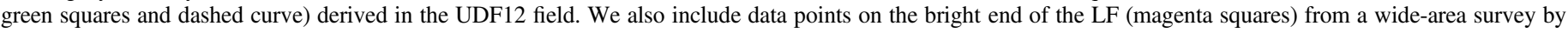
Bowler et al. (2014).

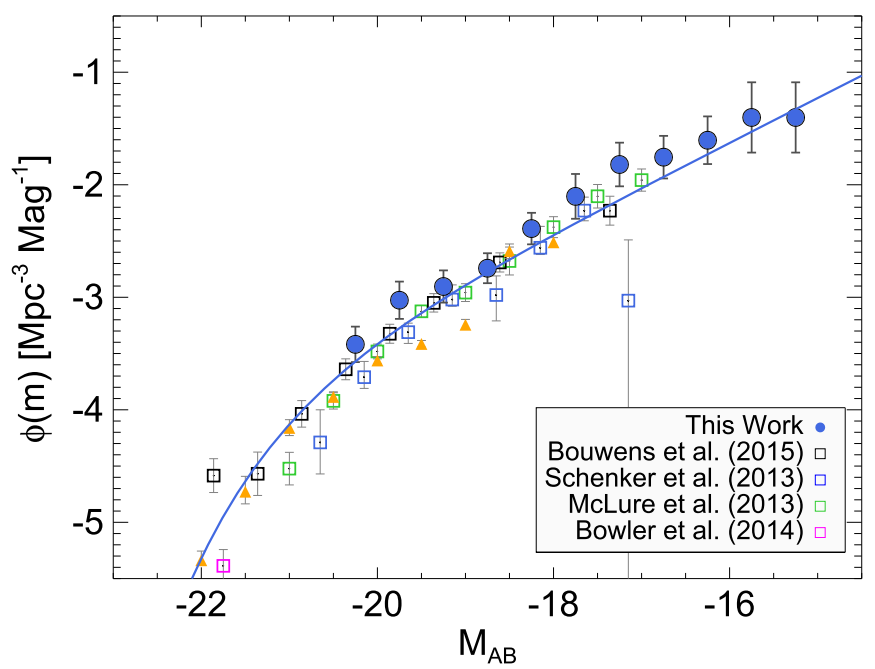

Figure 8. Combined constraints from the clusters and parallel fields on the UV LF at $z \sim 7$. The color code for the data points and the best-fit Schechter function are the same as in Figure 7.

Several theoretical models and cosmological simulations produce predictions for the UV LF at high redshift. For instance, our LF results at $z \sim 7$ are in good agreement with the hydrodynamical simulations of Jaacks et al. (2012) with $M^{\star}=-20.82$ and $\log \left(\phi^{\star}\right)=-3.74$, although they predict a steeper faint-end slope of $\alpha=-2.30$ down to a similar lower magnitude limit of $M_{\mathrm{UV}}=-15$. Theoretical models by Mason et al. (2015) find a closer slope of $\alpha=-1.95 \pm 0.17$ down to a magnitude limit of $M_{\mathrm{UV}}=-12$, corresponding to a halo mass of $10^{9} M_{\odot}$. Similarly, Kimm \& Cen (2014) find a theoretical faint magnitude limit of $M_{\mathrm{UV}}=-13$ with a faint-end slope of $\alpha=-1.9$. Semianalytical models of Dayal et al. (2014) also find a steep faint-end slope of $\alpha \sim-2.02$ at $z \sim 7$, in good agreement with our observations.

\subsection{The $U V L F$ at $z \sim 8$}

Regarding the redshift $z \sim 8 \mathrm{LF}$, we followed the same procedure used for the $z \sim 7 \mathrm{LF}$. However, in lensing clusters, the survey volume at $z \sim 8$ is much more significantly reduced than at $z \sim 7$. In the extreme case of MACS 0717, the total survey volume is about $460 \mathrm{Mpc}^{3}$, whereas it reaches $3600 \mathrm{Mpc}^{3}$ in $\mathrm{A} 2744$. Therefore, we expect lower galaxy number counts at $z \sim 8$ for high magnification values. In total, we detect only two galaxies with intrinsic magnitudes fainter than $M_{\mathrm{UV}}=-18$, where the uncertainties on the LF estimate are very large (see Figure 10). Unlike the $z \sim 7 \mathrm{LF}$, we do not have strong constraints on the faint end of the LF at $z \sim 8$. At brighter magnitudes the LF is better constrained thanks to the addition of the parallel fields and appears in agreement with previous results in the literature. As discussed in Atek et al. (2015), the redshift 8 galaxy selection in A2744 clearly shows an overdensity (see also Zheng et al. 2014; Ishigaki et al. 2015a), which translates into a large excess in the UV LF. Here we decided not to exclude the entire A2744 field in 

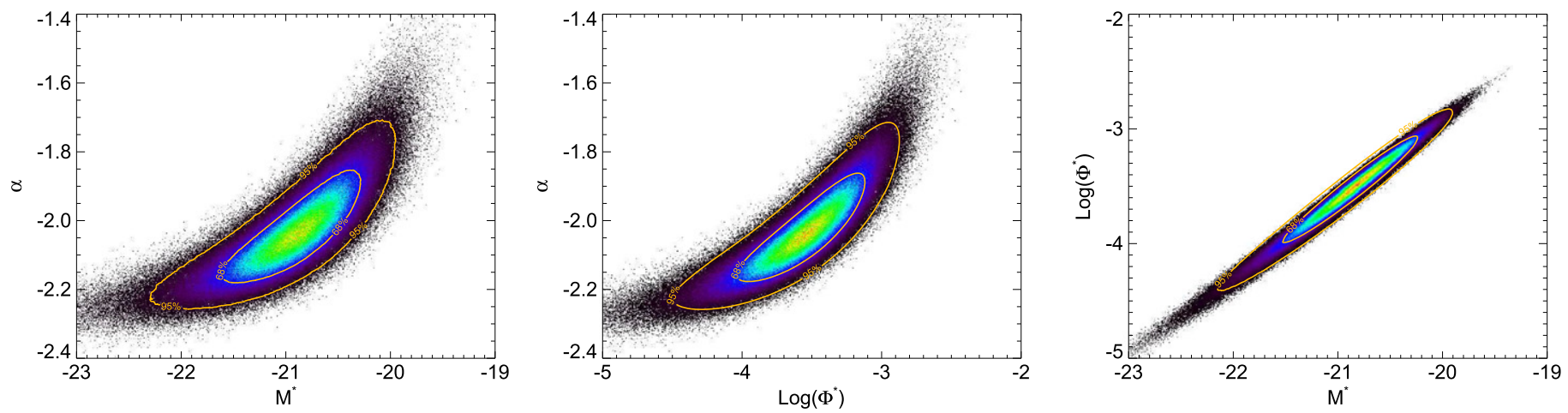

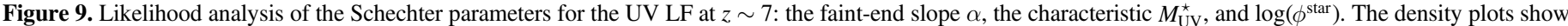

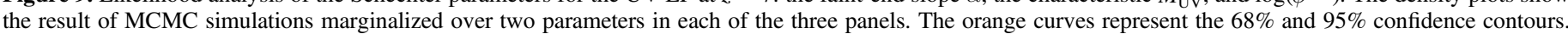

Table 5

Comparison of the Best-fit $z \sim 7$ Schechter Parameters

\begin{tabular}{lccc}
\hline \hline Reference & $\begin{array}{c}M_{\mathrm{UV}}^{\star} \\
(\mathrm{AB} \text { mag })\end{array}$ & $\alpha$ & $\begin{array}{c}\log _{10} \phi^{\star} \\
\left(\mathrm{Mpc}^{-3}\right)\end{array}$ \\
\hline This work & $-20.89_{-0.72}^{+0.60}$ & $-2.04_{-0.13}^{+0.17}$ & $-3.54_{-0.45}^{+0.48}$ \\
Atek et al. (2015) & $-20.90_{-0.73}^{+0.90}$ & $-2.01_{-0.28}^{+0.20}$ & $-3.55_{-0.57}^{+0.57}$ \\
Ishigaki et al. (2015a) $^{\mathrm{a}}$ & $-20.45_{-0.2}^{+0.1}$ & $-1.94_{-0.10}^{+0.09}$ & $-3.30_{-0.20}^{+0.10}$ \\
Bouwens et al. (2015b) & $-21.04 \pm 0.26$ & $-2.06 \pm 0.12$ & $-3.65_{-0.17}^{+0.27}$ \\
Finkelstein et al. (2014) & $-21.03_{-0.50}^{+0.37}$ & $-2.03_{-0.20}^{+0.21}$ & $-3.80_{-0.26}^{+0.41}$ \\
McLure et al. (2013) & $-19.90_{-0.28}^{+0.23}$ & $-1.90_{-0.15}^{+0.14}$ & $-3.35_{-0.45}^{+0.28}$ \\
\hline
\end{tabular}

Note.

${ }^{\mathrm{a}}$ Using the first HFF cluster A2744.

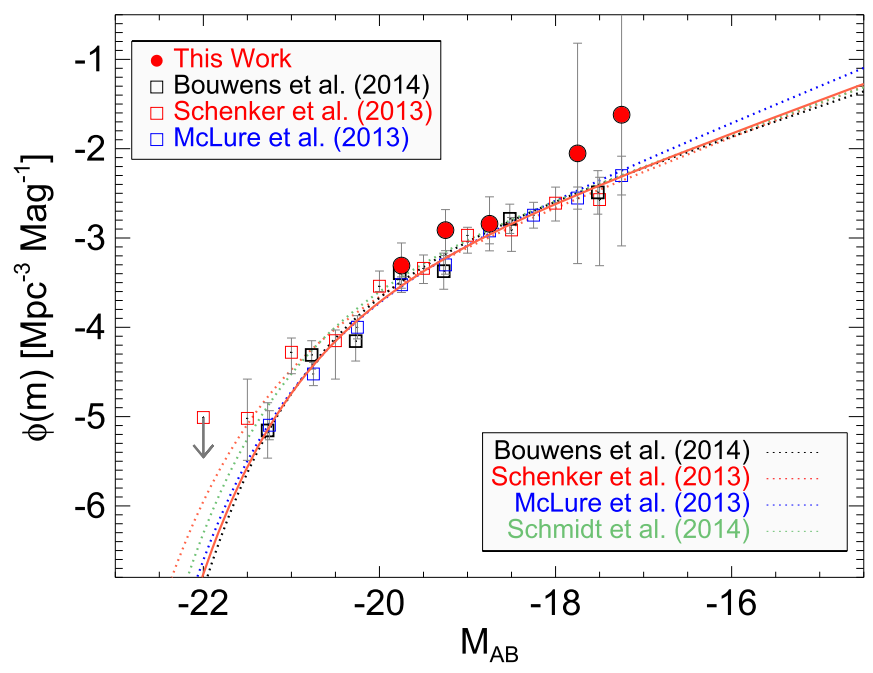

Figure 10. Combined constraints from the clusters and parallel fields on the UV LF at $z \sim 8$. The red circles represent our LF determination, while the red squares are taken from Schenker et al. (2013a), the black squares from Bouwens et al. (2015b), and the blue squares from McLure et al. (2013). The green curve is the unbinned UV LF of Schmidt et al. (2014). The best Schechter fits from the same literature results are also shown with dotted lines (see legend in the inset).

the combined LF to avoid introducing a well-known bias due to cosmic variance (see also Ishigaki et al. 2015b).

\subsection{Implications for Cosmic Reionization}

In this study, we reliably extend the UV LF to unprecedented depth and put strong constraints on the faint-end slope down to
$M_{\mathrm{UV}}=-15.25$. The most important result is that the faintend slope remains very steep down to a luminosity of $0.005 L^{\star}$, the characteristic UV luminosity at $z \sim 7$. Also, thanks to the combination of six fields, we have significantly reduced the uncertainties on the faint-end slope to about $5 \%$. With these strong constraints in hand, we can now integrate the UV LF to derive the UV luminosity density at $z \sim 7$. The main advantage of these observations is the ability to set the integration lower limit to $M_{\mathrm{UV}}=-15$. Unlike previous results that extrapolate the UV LF to lower magnitude, we use an observational constraint to estimate the ultraviolet photon budget from galaxies. For instance, based on the UV LF results in blank fields, Bouwens et al. (2015b) report a total UV luminosity density of $\log \left(\rho_{\mathrm{UV}}\right)=25.98 \pm 0.06 \mathrm{erg} \mathrm{s}^{-1} \mathrm{~Hz}^{-1} \mathrm{Mpc}^{-3}$ and Finkelstein et al. (2014) a value of $\log \left(\rho_{\mathrm{UV}}\right)=$ $25.77 \pm 0.06 \mathrm{erg} \mathrm{s}^{-1} \mathrm{~Hz}^{-1} \mathrm{Mpc}^{-3}$ at $z \sim 7$. Both values are computed down to $M_{\mathrm{UV}}=-17 \mathrm{AB}$, which is the limiting magnitude of their observations. Here we compute the UV luminosity density down to $M_{\mathrm{UV}}=-15$ and find $\log \left(\rho_{\mathrm{UV}}\right)=26.2 \pm 0.13 \mathrm{erg} \mathrm{s}^{-1} \mathrm{~Hz}^{-1} \mathrm{Mpc}^{-3}$.

In order to determine whether the UV luminosity produced by galaxies is sufficient to reionize the IGM, one needs to estimate additional parameters, for which observational constraints remain challenging. First, the conversion factor from the UV luminosity to ionizing radiation $\xi_{\text {ion }}\left(\mathrm{erg} \mathrm{s}^{-1} \mathrm{~Hz}\right)$ depends on the star formation history of galaxies. The value of $\xi_{\text {ion }}$ is generally constrained using stellar population models of early galaxies and the observed UV slope $\beta$ of $z>6$ galaxies (Bolton \& Haehnelt 2007; Kuhlen \& Faucher-Giguère 2012; Robertson et al. 2015). Once the ionizing photon production is determined, we need to estimate its escape fraction $f_{\text {esc }}$ from galaxies to ionize the IGM. Direct observational constraints of $f_{\text {esc }}$ are difficult, especially at $z>6$ because of the high opacity of the intervening hydrogen residuals on the line of sight. Many studies and deep surveys were dedicated to the search of ionizing continuum (Lyman continuum) escape from $z<4$ galaxies. Very few detections were reported, however, and they show very low escape fractions relative to the UV radiation, of the order of a few percent (Shapley et al. 2006; Iwata et al. 2009; Siana et al. 2010; Nestor et al. 2013). More recently, de Barros et al. (2015) reported a spectroscopic detection of Lyman continuum emission in a $z \sim 3.2$ galaxy with a relative escape fraction ${ }^{16}$ of $f_{\text {esc }} \sim 65 \%$.

\footnotetext{
16 The relative escape fraction is the ratio between the fraction of escaping Lyman continuum photons and the fraction of escaping photons at $1500 \AA$ (Steidel et al. 2001).
} 


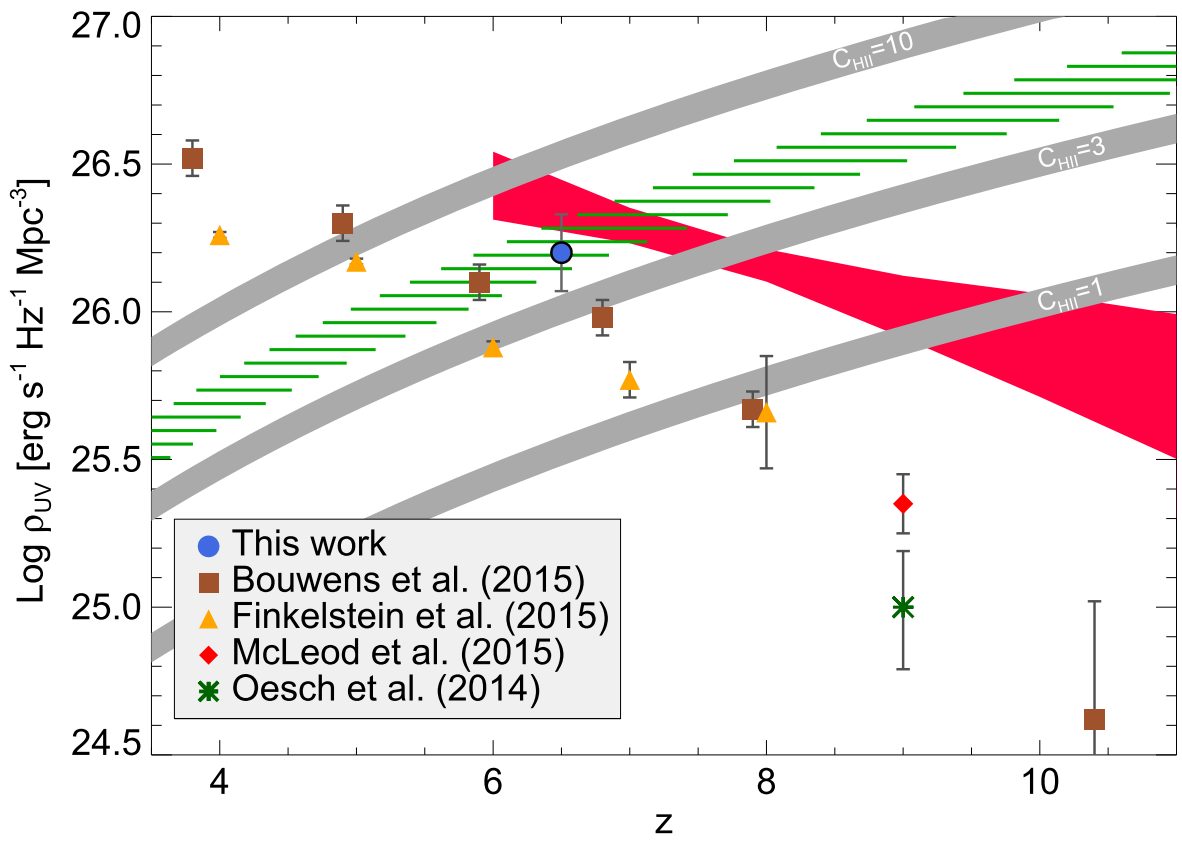

Figure 11. Evolution of the UV luminosity density of galaxies with redshift. Our determination (blue circle) is based on the integration of the UV LF down to $M_{\mathrm{UV}}=-15$. The brown squares represent the most recent results of Bouwens et al. (2015b), who integrate the UV LF down to $M_{\mathrm{UV}}=-17$. The orange triangles are the result of Finkelstein et al. (2014), who integrate to a conservative limit of $M_{\mathrm{UV}}=-18$. The red diamond and the green asterisk are the UV luminosity densities derived at $z \sim 9$ by McLeod et al. (2015) and Oesch et al. (2014), respectively. Both studies integrate the UV LF down to an observational limit of $M_{\mathrm{UV}}=-17.7$, which corresponds to a star formation rate of $\mathrm{SFR}_{\lim }=0.7 M_{\odot} \mathrm{yr}^{-1}$. All the error bars indicate $1 \sigma$ uncertainties. The shaded gray region shows the UV luminosity density based on the ionizing emissivity (Madau et al. 1999) required to maintain the IGM ionized at a certain redshift and for an escape fraction of ionizing radiation of $f_{\text {esc }} \sim 20 \%$ and three different values for the clumping factor $C_{\mathrm{H} \text { II }}=1,3$, and 10 . The red shaded region shows the $68 \%$ confidence interval for the evolution of the galaxy UV luminosity density based on constraints on the ionizing emissivity of Bouwens et al. (2015a). The green hatched region corresponds to the UV luminosity density required to maintain the IGM ionized for a clumping factor of $C_{\mathrm{H} \mathrm{II}}=3$ and escape fraction in the range $f_{\text {esc }}=10 \%-15 \%$, which encompasses our value at $z \sim 7$.

In Figure 11, we show our determination of the UV luminosity density together with the most recent results in the literature as a function of redshift, including Bouwens et al. (2015b) and Finkelstein et al. (2014) at $z=4-8$, Bouwens et al. (2015b) at $z=10$, and McLeod et al. (2015) and Oesch et al. (2014) at $z \sim 9$. While their values are based on the integration of the UV LF down to a magnitude limit between $M_{\mathrm{UV}}=-18$ and $M_{\mathrm{UV}}=-17$, our integration limit is two magnitudes fainter at $M_{\mathrm{UV}}=-15$. There is a significant difference between the results of Bouwens et al. (2015b) and Finkelstein et al. (2014) owing to the fact that the UV LF in the former study extends one magnitude deeper than the latter. Finkelstein et al. (2014) did not make use of the full IR data available in the HUDF, which also explains the larger uncertainties in their faint-end slope constraints. The UV luminosity density of galaxies at $z \sim 7$ determined in the present work is clearly larger than previous determinations, owing to a steep faint-end slope and a very faint integration limit. We can now assess whether this UV production is sufficient to ionize the IGM. We use the photon emission rate per unit cosmological comoving volume required to maintain reionization at a given redshift determined in Madau et al. (1999):

$$
\dot{N}(z)=\left(10^{51.2} \mathrm{~s}^{-1} \mathrm{Mpc}^{-3}\right) C_{30}\left(\frac{1+z}{6}\right)^{3}\left(\frac{\Omega_{b} h^{2}}{0.02}\right)^{2}
$$

where $C_{30}$ is the clumping factor $C_{\mathrm{H} \text { II }}=\left\langle n_{\mathrm{H} \text { II }}^{2}\right\rangle /\left\langle n_{\mathrm{H} \mathrm{II}}\right\rangle^{2}$ normalized to $C_{\mathrm{H} \text { II }}=30$, and $n_{\mathrm{H} \text { II }}$ is the mean comoving hydrogen density in the universe. This is the minimum value for which the ionizing emission balances the recombination rate. We show in Figure 11 this limit, converted to UV luminosity density (see Madau et al. 1999; Bolton \& Haehnelt 2007) assuming an escape fraction of the ionizing radiation of $f_{\text {esc }}=20 \%$ and three different values for the clumping factor. Assuming a standard value of $C_{\mathrm{H} \text { II }}=3$ for the clumping factor of the IGM (Pawlik et al. 2009; Finlator et al. 2012), our determination of galaxy UV luminosity density at $z \sim 7$ is sufficient to maintain reionization. We also show that an escape fraction as low as $f_{\text {esc }}=10 \%-15 \%$ (green hatched region) is already sufficient to ionize the IGM at $z \sim 7$. At the same redshift, the ionizing emissivity constraints of Bouwens et al. (2015a) are close to our UV density constraints.

With current observations we are not able to put better constraints on the faint end of the UV LF at $z \sim 8$ and hence on the ionizing emissivity of galaxies at this redshift. The conclusions from the deep blank fields are still affected by large uncertainties that prevent any strong claims regarding the contribution of galaxies to the ionizing budget at $z \sim 8$ (Finkelstein et al. 2014; Bouwens et al. 2015b). Future HST observations of the remaining HFF clusters might add better constrains on the UV luminosity density at $z \sim 8$ with additional highly magnified faint galaxies.

\section{SUMMARY}

Combining HST observations of lensing clusters and parallel fields of the HFF program, we computed the galaxy UV LF between $z=6$ and $z=8$. We assembled a large sample of about 250 galaxy candidates in this redshift range using the Lyman break photometric selection. In the cluster cores, we corrected 
the deep HST images for ICL and bright cluster galaxy light using a median filtering before object detection, while performing the photometry in the original images. This technique helps the detection of faint galaxies contaminated by cluster light but does not increase significantly the number of galaxies in the cluster center because of the very small volume probed in those regions that have very high magnification.

1. Using the latest lensing models produced by the CATS team for the three clusters A2744 (Jauzac et al. 2015), MACS 0416 (Jauzac et al. 2014), and MACS 0717 (Limousin et al. 2015), we have performed completeness simulations in the source plane that take into account all lensing effects. Thanks to the lensing magnification, the completeness function extends down to fainter magnitudes in the cluster fields than in the parallels. On the other hand, the total survey volume goes from $\sim 4 \operatorname{arcmin}^{2}$ in the parallel fields down to $0.6-1 \operatorname{arcmin}^{2}$ in lensing fields.

2. We computed the UV LF for all individual fields and for the combined sample at $z \sim 7$. The lensing magnification allows us to extend the LF down to an absolute UV magnitude limit of $M_{\mathrm{UV}}=-15.25$, which is more than 2 mag deeper than any study of the deep blank fields. Most importantly, we show that the faint-end slope remains very steep at $\alpha=-2.04_{-0.17}^{+0.13}$ at such a faint intrinsic luminosity. When excluding the MACS 0717 cluster, the uncertainties on the faint-end slope decrease to $\sigma_{\alpha} \sim 0.1$. The best Schechter fit yields a characteristic magnitude of $M^{\star}=-20.89_{-0.72}^{+0.60}$ and $\log \left(\phi^{\star}\right)=-3.54_{-0.45}^{+0.48}$. This is in good agreement with most of the recent results in blank fields (Finkelstein et al. 2014; Bouwens et al. 2015b), which were limited to $M_{\mathrm{UV}}=-17.5$, and early results of Atek et al. (2015).

3. Our determination of the UV LF at $z \sim 8$ does not reach beyond $M_{\mathrm{UV}}=-18$ because we detect only two galaxies in the two fainter magnitude bins. Albeit with large uncertainties due to small number statistics and incompleteness uncertainties, the currently determined LF points confirm the results of previous studies.

4. On observational grounds, we integrate the UV LF down to a magnitude limit of $M_{\mathrm{UV}}=-15$ and find $\log$ $\left(\rho_{\mathrm{UV}}\right)=26.2 \pm 0.13 \mathrm{erg} \mathrm{s}^{-1} \mathrm{~Hz}^{-1} \mathrm{Mpc}^{-3}$. Assuming standard values for the ionizing conversion factor $\xi_{\text {ion }}$ and the clumping factor $C_{\mathrm{H} \text { II }}=3$, the ionizing budget of galaxies would be sufficient to maintain the IGM ionized by $z \sim 7$ provided that the ionizing escape fraction from galaxies is greater than $10 \%$. Future observations of the HFF program will allow us to decrease the uncertainties on the UV luminosity density at $z \sim 7$ and perhaps improve the constraints on the UV luminosity density at $z \sim 8$, which are mostly based on blank-field observations.

With the help of gravitational lensing, we have produced the best constraints currently available on the UV LF and the contribution of galaxies to the IGM reionization at $z \sim 7$. We clearly demonstrate here the great potential and the feasibility of peering into the early universe through these cosmic telescopes. The remaining HFF clusters will certainly help improve even more our constraints on the UV LF at $z \sim 7$ and in particular at $z \sim 8$ to better assess the role of galaxies in the cosmic reionization process. Our results also show the great promise of future programs targeting lensing fields and pave the way to observing programs with the James Webb Space Telescope or the Wide-Field Infrared Survey Telescope, which are scheduled for launch in the near future.

We thank the anonymous referee for important comments that improved the manuscript, and Pascal Oesch and Rychard Bouwens for useful discussions. We want to thank the STScI and the HFF team for their efforts in obtaining and reducing the HST data. H.A. and J.-P.K. are supported by the European Research Council (ERC) advanced grant "Light on the Dark" (LIDA). J.R. acknowledges support from the ERC starting grant CALENDS. M.J. acknowledges support from the Leverhulme Trust (grant number PLP-2011-003) and Science and Technology Facilities Council (grant number ST/ L00075X/1). P.N. acknowledges support from NSF theory grant AST-1044455 and a theory grant from Space Telescope Science Institute HST-AR1214401. M.L. acknowledges support from CNRS.

\section{REFERENCES}

Abell, G. O., Corwin, H. G., Jr., \& Olowin, R. P. 1989, ApJS, 70, 1 Arnouts, S., Schiminovich, D., Ilbert, O., et al. 2005, ApJL, 619, L43 Atek, H., Kneib, J.-P., Pacifici, C., et al. 2014a, ApJ, 789, 96 Atek, H., Richard, J., Kneib, J.-P., et al. 2014b, ApJ, 786, 60 Atek, H., Richard, J., Kneib, J.-P., et al. 2015, ApJ, 800, 18 Atek, H., Schaerer, D., \& Kunth, D. 2009, A\&A, 502, 791

Atek, H., Siana, B., Scarlata, C., et al. 2011, ApJ, 743, 121

Beckwith, S. V. W., et al. 2006, AJ, 132, 1729

Bennett, C. L., et al. 2013, ApJS, 208, 20

Bertin, E., \& Arnouts, S. 1996, A\&AS, 117, 393

Bolton, J. S., \& Haehnelt, M. G. 2007, MNRAS, 382, 325

Bouwens, R., et al. 2012, arXiv

Bouwens, R. J., Illingworth, G. D., Blakeslee, J. P., Broadhurst, T. J., \& Franx, M. 2004, ApJL, 611, L1

Bouwens, R. J., Illingworth, G. D., Blakeslee, J. P., \& Franx, M. 2006, ApJ, 653,53

Bouwens, R. J., Illingworth, G. D., Oesch, P. A., et al. 2015a, arXiv Bouwens, R. J., Illingworth, G. D., Oesch, P. A., et al. 2015b, ApJ, 803, 34 Bowler, R. A. A., Dunlop, J. S., McLure, R. J., et al. 2014, MNRAS, 440, 2810 Bradač, M., Schneider, P., Lombardi, M., \& Erben, T. 2005, A\&A, 437, 39 Bradley, L. D., Bouwens, R. J., Zitrin, A., et al. 2012, ApJ, 747, 3 Bradley, L. D., Zitrin, A., Coe, D., et al. 2014, ApJ, 792, 76

Bunker, A. J., Stanway, E. R., Ellis, R. S., \& McMahon, R. G. 2004, MNRAS, 355,374

Bunker, A. J., Wilkins, S., Ellis, R. S., et al. 2010, MNRAS, 409, 855

Caruana, J., Bunker, A. J., Wilkins, S. M., et al. 2014, MNRAS, 443, 2831

Casertano, S., de Mello, D., Dickinson, M., et al. 2000, AJ, 120, 2747

Chabrier, G., Baraffe, I., Allard, F., \& Hauschildt, P. 2000, ApJ, 542, 464

Chornock, R., Berger, E., Fox, D. B., et al. 2014, arXiv

Coe, D., Bradley, L., \& Zitrin, A. 2015, ApJ, 800, 84

Coe, D., Zitrin, A., Carrasco, M., et al. 2013, ApJ, 762, 32

Coleman, G. D., Wu, C.-C., \& Weedman, D. W. 1980, ApJS, 43, 393

Dayal, P., Ferrara, A., Dunlop, J. S., \& Pacucci, F. 2014, MNRAS, 445, 2545 de Barros, S., Vanzella, E., Amorín, R., et al. 2015, arXiv:1507.06648

Diego, J. M., Broadhurst, T., Molnar, S. M., Lam, D., \& Lim, J. 2014, arXiv Duncan, K., \& Conselice, C. J. 2015, MNRAS, 451, 2030

Ebeling, H., Barrett, E., Donovan, D., et al. 2007, ApJL, 661, L33

Ebeling, H., Edge, A. C., \& Henry, J. P. 2001, ApJ, 553, 668

Ebeling, H., Edge, A. C., Mantz, A., et al. 2010, MNRAS, 407, 83

Ebeling, H., Ma, C.-J., \& Barrett, E. 2014, ApJS, 211, 21

Elíasdóttir, Á., Limousin, M., Richard, J., et al. 2007, arXiv:0710.5636

Fan, X., Strauss, M. A., Becker, R. H., et al. 2006, AJ, 132, 117

Ferguson, H. C., Dickinson, M., Giavalisco, M., et al. 2004, ApJL, 600, L107

Finkelstein, S. L., Ryan, Jr., R. E., Papovich, C., et al. 2014, arXiv:1410.5439 Finlator, K., Oh, S. P., Özel, F., \& Davé, R. 2012, MNRAS, 427, 2464 Giavalisco, M. 2002, ARA\&A, 40, 579

Grazian, A., Castellano, M., Koekemoer, A. M., et al. 2011, A\&A, 532, A33 Grazian, A., Castellano, M., Fontana, A., et al. 2012, A\&A, 547, A51

Grillo, C., Suyu, S. H., Rosati, P., et al. 2015, ApJ, 800, 38 
Hathi, N. P., Jansen, R. A., Windhorst, R. A., et al. 2008, AJ, 135, 156

Hayes, M., Laporte, N., Pelló, R., Schaerer, D., \& Le Borgne, J.-F. 2012, MNRAS, 425, L19

Huang, K.-H., Ferguson, H. C., Ravindranath, S., \& Su, J. 2013, ApJ, 765, 68

Huang, X., Zheng, W., Wang, J., et al. 2015, ApJ, 801, 12

Ishigaki, M., Kawamata, R., Ouchi, M., et al. 2015a, ApJ, 799, 12

Ishigaki, M., Ouchi, M., \& Harikane, Y. 2015b, arXiv

Iwata, I., Inoue, A. K., Matsuda, Y., et al. 2009, ApJ, 692, 1287

Jaacks, J., Choi, J.-H., Nagamine, K., Thompson, R., \& Varghese, S. 2012, MNRAS, 420, 1606

Jauzac, M., Clément, B., Limousin, M., et al. 2014, MNRAS, 443, 1549

Jauzac, M., Richard, J., Jullo, E., et al. 2015, MNRAS, 452, 1437

Johnson, T. L., Sharon, K., Bayliss, M. B., et al. 2014, arXiv

Jullo, E., \& Kneib, J.-P. 2009, MNRAS, 395, 1319

Jullo, E., Kneib, J.-P., Limousin, M., et al. 2007, NJPh, 9, 447

Karman, W., Caputi, K. I., Grillo, C., et al. 2015, A\&A, 574, A11

Kawamata, R., Ishigaki, M., Shimasaku, K., Oguri, M., \& Ouchi, M. 2015, ApJ, 804, 103

Kimm, T., \& Cen, R. 2014, ApJ, 788, 121

Kinney, A. L., Calzetti, D., Bohlin, R. C., et al. 1996, ApJ, 467, 38

Kneib, J.-P. 1993, PhD thesis, Univ. Paul Sabatier

Kneib, J.-P., \& Natarajan, P. 2011, A\&ARv, 19, 47

Krist, J. E., Hook, R. N., \& Stoehr, F. 2011, Proc. SPIE, 8127, 81270J

Kuhlen, M., \& Faucher-Giguère, C.-A. 2012, MNRAS, 423, 862

Madau, P., Haardt, F., \& Rees, M. J. 1999, ApJ, 514, 648

Mann, A. W., \& Ebeling, H. 2012, MNRAS, 420, 2120

Mason, C., Trenti, M., \& Treu, T. 2015, arXiv

McLeod, D. J., McLure, R. J., Dunlop, J. S., et al. 2015, MNRAS, 450, 3032

McLure, R. J., Cirasuolo, M., Dunlop, J. S., Foucaud, S., \& Almaini, O. 2009, MNRAS, 395, 2196

McLure, R. J., Dunlop, J. S., Bowler, R. A. A., et al. 2013, MNRAS, 432, 2696

Medezinski, E., Umetsu, K., Okabe, N., et al. 2015, arXiv

Merten, J., Coe, D., Dupke, R., et al. 2011, MNRAS, 417, 333

Mitchell-Wynne, K., Cooray, A., Gong, Y., et al. 2015, arXiv:1509.02935

Montes, M., \& Trujillo, I. 2014, ApJ, 794, 137

Mosleh, M., Williams, R. J., Franx, M., et al. 2012, ApJL, 756, L12

Nestor, D. B., Shapley, A. E., Kornei, K. A., Steidel, C. C., \& Siana, B. 2013, ApJ, 765, 47

Oesch, P. A., Bouwens, R. J., Illingworth, G. D., et al. 2010, ApJL, 709, L16

Oesch, P. A., Bouwens, R. J., Illingworth, G. D., et al. 2012, ApJ, 745, 110

Oesch, P. A., Bouwens, R. J., Illingworth, G. D., et al. 2014, ApJ, 786, 108

Ogrean, G., van Weeren, R., Jones, C., et al. 2015, arXiv:1505.05560

Ono, Y., Ouchi, M., Curtis-Lake, E., et al. 2013, ApJ, 777, 155

Ouchi, M., Mobasher, B., Shimasaku, K., et al. 2009, ApJ, 706, 1136

Owers, M. S., Randall, S. W., Nulsen, P. E. J., et al. 2011, ApJ, 728, 27

Pawlik, A. H., Schaye, J., \& van Scherpenzeel, E. 2009, MNRAS, 394, 1812

Pénin, A., Cuby, J.-G., Clément, B., et al. 2015, A\&A, 577, A74

Pentericci, L., Vanzella, E., Fontana, A., et al. 2014, ApJ, 793, 113
Planck Collaboration Ade, P. A. R., Aghanim, N., et al. 2015, arXiv: 1502.01589

Postman, M., Coe, D., Benítez, N., et al. 2012, ApJS, 199, 25

Rawle, T. D., Altieri, B., Egami, E., et al. 2015, arXiv:1508.00586

Reddy, N. A., \& Steidel, C. C. 2009, ApJ, 692, 778

Richard, J., Jauzac, M., Limousin, M., et al. 2014, arXiv:1405.3303

Richard, J., Patricio, V., Martinez, J., et al. 2015, MNRAS, 446, L16

Robertson, B. E., Ellis, R. S., Dunlop, J. S., et al. 2014, arXiv

Robertson, B. E., Ellis, R. S., Furlanetto, S. R., \& Dunlop, J. S. 2015, ApJL, 802, L19

Rodney, S. A., Patel, B., Scolnic, D., et al. 2015, arXiv:1505.06211

Schaerer, D., \& de Barros, S. 2009, A\&A, 502, 423

Schechter, P. 1976, ApJ, 203, 297

Schenker, M. A., Ellis, R. S., Konidaris, N. P., \& Stark, D. P. 2013a, ApJ, 777,67

Schenker, M. A., Ellis, R. S., Konidaris, N. P., \& Stark, D. P. 2014, ApJ, 795,20

Schenker, M. A., Robertson, B. E., Ellis, R. S., et al. 2013b, ApJ, 768, 196

Schiminovich, D., Ilbert, O., Arnouts, S., et al. 2005, ApJL, 619, L47

Schirmer, M., Carrasco, E. R., Pessev, P., et al. 2015, ApJS, 217, 33

Schmidt, K. B., Treu, T., Trenti, M., et al. 2014, ApJ, 786, 57

Shapley, A. E., Steidel, C. C., Pettini, M., Adelberger, K. L., \& Erb, D. K. 2006, ApJ, 651, 688

Siana, B., Teplitz, H. I., Ferguson, H. C., et al. 2010, ApJ, 723, 241

Soucail, G., Fort, B., Mellier, Y., \& Picat, J. P. 1987, A\&A, 172, L14

Stark, D. P., Ellis, R. S., Chiu, K., Ouchi, M., \& Bunker, A. 2010, MNRAS, 408, 1628

Steidel, C. C., Giavalisco, M., Pettini, M., Dickinson, M., \& Adelberger, K. L. 1996, ApJL, 462, L17

Steidel, C. C., Pettini, M., \& Adelberger, K. L. 2001, ApJ, 546, 665

Trenti, M., Bradley, L. D., Stiavelli, M., et al. 2011, ApJL, 727, L39

Treu, T., Schmidt, K. B., Trenti, M., Bradley, L. D., \& Stiavelli, M. 2013, ApJL, 775, L29

Treu, T., Schmidt, K. B., Brammer, G. B., et al. 2015, arXiv:1509.00475

van der Wel, A., Straughn, A. N., Rix, H.-W., et al. 2011, ApJ, 742, 111

Vanzella, E., Giavalisco, M., Dickinson, M., et al. 2009, ApJ, 695, 1163

Wang, X., Hoag, A., Huang, K., et al. 2015, arXiv:1504.02405

Wilkins, S. M., Bunker, A. J., Ellis, R. S., et al. 2010, MNRAS, 403, 938

Wilkins, S. M., Coulton, W., Caruana, J., et al. 2013, MNRAS, 435, 2885

Willott, C. J., McLure, R. J., Hibon, P., et al. 2013, AJ, 145, 4

Wise, J. H., Demchenko, V. G., Halicek, M. T., et al. 2014, MNRAS, 442, 2560

Wong, K. C., Ammons, S. M., Keeton, C. R., \& Zabludoff, A. I. 2012, ApJ, 752, 104

Zheng, W., Shu, X., Moustakas, J., et al. 2014, arXiv:1402.6743

Zitrin, A., Broadhurst, T., Coe, D., et al. 2011, MNRAS, 413, 1753

Zitrin, A., Ellis, R. S., Belli, S., \& Stark, D. P. 2015, ApJL, 805, L7

Zitrin, A., Zheng, W., Broadhurst, T., et al. 2014, arXiv:1407.3769 